\title{
Regularity of the diffusion coefficient matrix for the lattice gas with energy
}

\author{
Yukio Nagahata \\ Department of Mathematical Science, Graduate School of Engineering Science, Osaka University, Toyonaka, 560-8531, Japan
}

Received 5 April 2003; received in revised form 4 November 2003; accepted 30 March 2004

Available online 11 September 2004

\begin{abstract}
In this paper we obtain the smoothness of the diffusion coefficient matrix for the lattice gas with energy. Furthermore we also obtain the smoothness of the central limit theorem variances for certain functions.

(c) 2004 Elsevier SAS. All rights reserved.
\end{abstract}

\section{Résumé}

Dans cet article, on montre la régularité de la matrice des coefficients de diffusion pour le gaz sur réseau avec énergie. On obtient également la régularité des variances associées à certaines fonctions par le théorème limite central.

(c) 2004 Elsevier SAS. All rights reserved.

\section{Introduction}

In our previous paper [6] we have introduced a lattice gas with energy and derived the fluctuation dissipation equation for it. In this paper we prove that the diffusion coefficient matrix appearing in the equation is smooth.

In the derivation of hydrodynamic limit, uniqueness of the Cauchy problem of the weak solution of limiting diffusion equation is needed. It seems unsolved in the existing literatures. But once smoothness and uniform ellipticity of the diffusion coefficient matrix is established and if there exists a Lipschitz continuous solution, then the uniqueness question is resolved.

The smoothness of the self-diffusion coefficient of the symmetric simple exclusion process is proved by Landim, Olla, and Varadhan [4], and the smoothness of the diffusion coefficient for a lattice gas reversible under the Bernoulli measures is proved by Bernardin [1].

It seems difficult to adapt to our model the method which introduced in [4] and developed in [1], since we do not have any suitable orthonormal basis (with respect to invariant measure) of functions on the configuration space.

E-mail address: nagahata@sigmath.es.osaka-u.ac.jp (Y. Nagahata). 
In this paper, we choose a basis of the space of continuous functions on the configuration space which is not orthonormal (with respect to invariant measure). We also introduce a Markov process whose state space is a set of indexes of the basis and which may be regarded as a dual process. By using this process, the diffusion coefficient matrix is given by a finite linear combination of smooth functions whose coefficient is given by expectation of the total occupation time for a certain infinite set. We prove that the expectation of the occupation time for the infinite set converges if $d \geqslant 3$, and diverges if $d=1,2$. But if we examine the linear combination more carefully, then we find that it is a difference of expectations of the total occupations times of a certain infinite set for the processes starting at two different points. Such difference makes sense as in the same way that makes the potential function of one or two dimensional random walk well defined.

This paper is organized as follows: In Section 2 we state the model and results. In Section 3 we introduce a basis of continuous functions on the configuration space and compute the coefficient of $L f$ with respect to the basis. We also introduce a Markov process which may be regarded as a dual process of our lattice gas, and solve the resolvent equation by using the process. In Section 4 we estimate the expectation of occupation time of certain set for the process in dimensions $d \geqslant 3$. In Section 5, we estimate an expectation of occupation time for a finite state continuous time stochastic jump process. In Section 6, we estimate an expectation of occupation time for certain set for the process. In Section 7 we prove the main results.

\section{Model and results}

Our lattice gas is a Markov process on the state space $X:=\{0,1, \ldots, k\}^{\mathbf{Z}^{d}}$. Let $\eta=\left(\eta_{x}\right)_{x \in \mathbf{Z}^{d}}$ stands for a generic element of $X$, so that for each $x, \eta_{x} \in\{0,1,2, \ldots, k\}$. For any local function $f$ we define operators $\pi^{(x, y)}$ and $\pi^{x \rightarrow y}$ by

$$
\begin{aligned}
& \pi^{(x, y)} f(\eta):=1_{\left\{\eta_{x} \neq 0, \eta_{y}=0\right\}}\left(f\left(\eta^{(x, y)}\right)-f(\eta)\right), \\
& \pi^{x \rightarrow y} f(\eta):=1_{\left\{\eta_{x} \geqslant 2,1 \leqslant \eta_{y} \leqslant k-1\right\}}\left(f\left(\eta^{x \rightarrow y}\right)-f(\eta)\right),
\end{aligned}
$$

where $\eta^{(x, y)}$ and $\eta^{x \rightarrow y}$ are the configurations defined by

$$
\begin{aligned}
\left(\eta^{(x, y)}\right)_{z} & := \begin{cases}\eta_{y}, & \text { if } z=x, \\
\eta_{x}, & \text { if } z=y, \\
\eta_{z}, & \text { otherwise },\end{cases} \\
\left(\eta^{x \rightarrow y}\right)_{z}: & = \begin{cases}\eta_{x}-1, & \text { if } z=x, \\
\eta_{y}+1, & \text { if } z=y, \\
\eta_{z}, & \text { otherwise. }\end{cases}
\end{aligned}
$$

For any local function $f$ we define the generator

$$
L f(\eta):=\sum_{x, y \in \mathbf{Z}^{d}:|x-y|=1}\left\{c_{\mathrm{ex}}\left(\eta_{x}\right) \pi^{(x, y)} f(\eta)+c_{\mathrm{ge}}\left(\eta_{x}\right) \pi^{x \rightarrow y} f(\eta)\right\}
$$

where $|\cdot|$ is the Euclidean norm of $\mathbf{Z}^{d}$ and $c_{\mathrm{ex}}(r), c_{\mathrm{ge}}(r)$ are positive functions of $r=0,1,2, \ldots, k$. Furthermore we suppose that $c_{\mathrm{ex}}(0)=c_{\mathrm{ge}}(0)=c_{\mathrm{ge}}(1)=0$ and $c_{\mathrm{ex}}(r)>0$ for $1 \leqslant r \leqslant k$ and $c_{\mathrm{ge}}(r)>0$ for $2 \leqslant r \leqslant k$.

The process is thought to model a time evolution of the dynamics of a gas of particles having energy. The values of $\eta_{x}$ are interpreted in such a way that if $\eta_{x}=0$, then the site $x$ is vacant, and if $\eta_{x} \neq 0$, then there exists a particle having (discrete) energy $\eta_{x}$ at site $x$. A particle at site $x$ moves to a nearest neighbor site $y$ at rate $c_{\mathrm{ex}}\left(\eta_{x}\right)$ if $y$ is vacant. One unit of energy of the particle at site $x$ is transferred to the particle at a neighboring site $y$ at rate $c_{\text {ge }}\left(\eta_{x}\right)$ if the energy of the particle at the site $y$ is less than $k$. 
Consider the family of product measures on the product space $\{0,1,2, \ldots, k\}^{\mathbf{Z}^{d}}$ with the marginal distribution

$$
v_{p, \alpha}\left(\left\{\eta: \eta_{x}=l\right\}\right):= \begin{cases}1-p & \text { if } l=0, \\ p \frac{1}{Z_{\alpha}} & \text { if } l=1, \\ p \frac{1}{Z_{\alpha}} \frac{\alpha_{\mathrm{ge}}(2) c_{\mathrm{ge}}(3) \cdots c_{\mathrm{ge}}(l)}{l-1} & \text { if } 2 \leqslant l \leqslant k\end{cases}
$$

for all $x \in \mathbf{Z}^{d}$, where $0 \leqslant p \leqslant 1,0 \leqslant \alpha<\infty$ and $Z_{\alpha}$ is the normalization constant. Put

$$
\rho:=\rho(p, \alpha):=E^{v_{p, \alpha}}\left[\eta_{0}\right],
$$

then $\rho$ is a rational function of two variables $p, \alpha$, and for each $p, \rho$ is a strictly increasing function of $\alpha$. Therefore there exists an inverse function $\tilde{\alpha}=\tilde{\alpha}(p, \rho)$ say. We define $P_{p, \rho}$ by

$$
P_{p, \rho}(\cdot):=v_{p, \tilde{\alpha}(p, \rho)}(\cdot),
$$

for $0 \leqslant p \leqslant 1$ and $p \leqslant \rho<k p$. For any local function $f, E_{p, \rho}[f]$ is a smooth function of $p$ and $\rho$. We can easily check that $L$ is symmetric with respect to $P_{p, \rho}$. One can show that there exists a unique closed extension of $L$ in the space of continuous functions $C(X)$ with supremum norm (see [5]). We denote by $e^{t L}$ the semigroup generated by the closed extension.

We define the shift operator $\tau_{x}$ for $x \in \mathbf{Z}^{d}$, which acts on all $A \subset \mathbf{Z}^{d}$, and local functions $f$ as well as configurations $\eta$ as follows:

$$
\begin{aligned}
& \tau_{x} A:=x+A, \\
& \tau_{x} f(\eta):=f\left(\tau_{x} \eta\right), \\
& \left(\tau_{x} \eta\right)_{z}:=\eta_{z-x} .
\end{aligned}
$$

Let $\tilde{D}(p, \rho)=\left(\tilde{D}_{i, j}(p, \rho)\right)_{i, j \in\{1,2\}}$ be a $2 \times 2$ symmetric matrix defined via the variational formula

$$
\begin{aligned}
(a \cdot \tilde{D}(p, \rho) a)= & \sum_{i, j} a_{i} \tilde{D}_{i, j}(p, \rho) a_{j} \\
:= & \inf _{u} E_{p, \rho}\left[c_{\mathrm{ex}}\left(\eta_{0}\right)\left\{\pi^{(0, e)}\left(a_{1} \eta_{0}+a_{2} 1_{\left\{\eta_{0} \neq 0\right\}}\right)+\sum_{x} \pi^{(0, e)} \tau_{x} u\right\}^{2}\right. \\
& \left.+c_{\mathrm{ge}}\left(\eta_{0}\right)\left\{\pi^{0 \rightarrow e}\left(a_{1} \eta_{0}+a_{2} 1_{\left\{\eta_{0} \neq 0\right\}}\right)+\sum_{x} \pi^{0 \rightarrow e} \tau_{x} u\right\}^{2}\right],
\end{aligned}
$$

where $a$ is any 2-dimensional vector, $e$ is a unit vector and $\inf _{u}$ is taken over all local functions. Put

$$
\begin{aligned}
& \chi(p, \rho):=\left(\begin{array}{cc}
E_{p, \rho}\left[\eta_{0}^{2}\right]-\rho^{2} & (1-p) \rho \\
(1-p) \rho & p(1-p)
\end{array}\right), \\
& D(p, \rho)=\tilde{D}(p, \rho) \chi^{-1}(p, \rho),
\end{aligned}
$$

for $0<p<1$ and $p<\rho<k p$. $\left(\chi^{-1}(p, \rho)\right.$ denotes the inverse matrix of $\chi(p, \rho)$.) Using these notations we can state our main result.

Theorem 2.1. Let $(p, \rho)$ such that $0<p<1$ and $p<\rho<k p$. Then the diffusion coefficient matrix for this model defined by (2) is a smooth function of $p$ and $\rho$, and has the smooth extension up to the boundary.

The smoothness of the diffusion coefficient matrix is implied by that of the central limit theorem variance for certain functions.

We define the currents $w_{e}^{E}, w_{e}^{P}$ by 


$$
\begin{aligned}
w_{e}^{E}(\eta):= & c_{\mathrm{ex}}\left(\eta_{0}\right) \eta_{0} 1_{\left\{\eta_{0} \neq 0, \eta_{1}=0\right\}}(\eta)-c_{\mathrm{ex}}\left(\eta_{1}\right) \eta_{1} 1_{\left\{\eta_{1} \neq 0, \eta_{0}=0\right\}}(\eta) \\
& +c_{\mathrm{ge}}\left(\eta_{0}\right) 1_{\left\{\eta_{0} \geqslant 2,1 \leqslant \eta_{1} \leqslant k-1\right\}}(\eta)-c_{\mathrm{ge}}\left(\eta_{1}\right) 1_{\left\{\eta_{1} \geqslant 2,1 \leqslant \eta_{0} \leqslant k-1\right\}}(\eta), \\
w_{e}^{P}(\eta)= & c_{\mathrm{ex}}\left(\eta_{0}\right) 1_{\left\{\eta_{0} \neq 0, \eta_{1}=0\right\}}(\eta)-c_{\mathrm{ex}}\left(\eta_{1}\right) 1_{\left\{\eta_{1} \neq 0, \eta_{0}=0\right\}}(\eta) .
\end{aligned}
$$

Denoted by $\mathcal{W}$ the linear space spanned by currents $w_{e}^{E}, w_{e}^{P}$ for all positive unit vectors $e$.

Theorem 2.2. Suppose $f \in \mathcal{W}$. Then $\int_{0}^{\infty} \sum_{x \in \mathbf{Z}^{d}} E_{p, \rho}\left[f \mathrm{e}^{L t} \tau_{x} f\right] \mathrm{d} t$ is a smooth function of $p$ and $\rho$.

Remark. If the dimensions $d$ is greater than or equal to 3, the present method is applicable to generalized exclusion process which is introduced by Kipnis, Landim and Olla [2], namely Theorems 2.1 and 2.2 are valid for it in dimensions $d \geqslant 3$.

\section{Basis of $C(X)$ and dual process}

In this section we will introduce a basis of the space of continuous functions on the configuration space and compute the coefficient of $L f$ with respect to the basis for a local function $f$.

Let $C(X)$ denote the space of continuous functions on $X$ with supremum norm. It is convenient to define a subspace $C_{0}(X)$ of $C(X)$ as follows: Let $C_{0}(X)$ be the set of functions such that there exist positive constants $c_{1}$ and $c_{2}$ with

$$
\sup _{\eta, \eta^{\prime}: \eta_{x}=\eta_{x}^{\prime} \text { for }|x|_{\infty} \leqslant l}\left|f(\eta)-f\left(\eta^{\prime}\right)\right| \leqslant c_{1} \mathrm{e}^{-c_{2} l}
$$

for all $l \geqslant 0$, where $|\cdot|_{\infty}$ is the supremum norm on $\mathbf{Z}^{d}$.

We define $\mathcal{A}$ by

$$
\mathcal{A}:=\left\{A=\left(A_{1}, \ldots, A_{k}\right): A_{i} \Subset \mathbf{Z}^{d}, \text { with } A_{i} \cap A_{j}=\emptyset \text { if } i \neq j\right\},
$$

where $A \Subset \mathbf{Z}^{d}$ means $A \subset \mathbf{Z}^{d}$ and $|A|$ is finite. For $A, B \in \mathcal{A}, B \subset A$ means $B_{i} \subset A_{i}$ for all $i, A \backslash B$ means that the $i$ th component is $A_{i} \backslash B_{i}$, and $\tau_{x} A$ means that $i$ th component is $\tau_{x} A_{i}(i=1,2, \ldots, k)$. We define two types of cardinality by

$$
\begin{aligned}
& \# A:=\sum_{i}\left|A_{i}\right|, \\
& \tilde{\#} A:=\sum_{i} i\left|A_{i}\right|,
\end{aligned}
$$

and a family of functions $\left\{\Psi_{A}\right\}_{A \in \mathcal{A}}$ by

$$
\Psi_{A}(\eta):=\prod_{i=1}^{k} \prod_{x \in A_{i}} 1_{\left\{\eta_{x}=i\right\}}(\eta) .
$$

For $A \in \mathcal{A}$ we define the special configuration $\eta^{A}$ by

$$
\left(\eta^{A}\right)_{z}:= \begin{cases}i & \text { if } z \in A_{i} \text { for } 1 \leqslant i \leqslant k, \\ 0 & \text { if } z \notin \bigcup_{i} A_{i} .\end{cases}
$$

The cardinality \#A and $\# A$ equals the number of particles and total energy for the special configuration $\eta^{A}$, respectively. The function $\Psi_{A}$ is the indicator function of the set of configurations $\eta$ for which each site of $\bigcup_{i} A_{i}$ is occupied by a particle and each particle on $A_{i}$ has the common energy $i$. 
Lemma 3.1. The family of functions $\left\{\Psi_{A}\right\}_{A \in \mathcal{A}}$ is a basis of the linear space $C(X)$. Furthermore, for $f \in C(X)$ if we define $\hat{f}: \mathcal{A} \rightarrow \mathbf{R}$ by

$$
\hat{f}(A):=\sum_{B \subset A}(-1)^{\#(A \backslash B)} f\left(\eta^{B}\right)
$$

then

$$
f(\eta)=\sum_{A \in \mathcal{A}} \hat{f}(A) \Psi_{A}(\eta)
$$

Proof. It is not difficult to see that $\left\{\Psi_{A}\right\}_{A \in \mathcal{A}}$ are linearly independent. Therefore we have only to prove (3).

Fix $A \in \mathcal{A}$ and $x \in \mathbf{Z}^{d}$ such that $x \notin \bigcup_{i} A_{i}$. For $1 \leqslant i \leqslant k$ and $B \subset A$, define $B^{x, i} \in \mathcal{A}$ by

$$
\left(B^{x, i}\right)_{j}:= \begin{cases}B_{i} \cup\{x\} & \text { if } j=i, \\ B_{j} & \text { if } j \neq i .\end{cases}
$$

Then for $f \in C(X)$,

$$
\hat{f}\left(A^{x, i}\right)=\sum_{B \subset A}(-1)^{\#(A \backslash B)+1} f\left(\eta^{B}\right)+\sum_{B \subset A}(-1)^{\#(A \backslash B)} f\left(\eta^{B^{x, i}}\right) .
$$

Let $f$ be a local function. Then there exists $\Lambda=\Lambda(f) \Subset \mathbf{Z}^{d}$ such that $f$ depends only on $\left\{\eta_{x}: x \in \Lambda\right\}$, and

$$
f\left(\eta^{A}\right)=f\left(\eta^{A \cap \Lambda}\right)
$$

is valid for all $A \in \mathcal{A}$, where $A \cap \Lambda \in \mathcal{A}$ is defined by $(A \cap \Lambda)_{i}:=A_{i} \cap \Lambda$. These two equality shows that

$$
\hat{f}(A)=0
$$

if $\bigcup_{i} A_{i} \cap \Lambda^{c} \neq \emptyset$. Therefore right-hand side of (3) is a finite sum. By (4), we have only to check the truth of

$$
f\left(\eta^{B}\right)=\sum_{A \in \mathcal{A}} \hat{f}(A) \Psi_{A}\left(\eta^{B}\right)
$$

for all $B \in \mathcal{A}$ such that $\bigcup B_{i} \subset \Lambda$. By the binomial expansion

$$
\sum_{A \subset B}(-1)^{\#(B \backslash A)}=(1-1)^{\# B}
$$

for $B \in \mathcal{A}$, it holds that

$$
\sum_{A \in \mathcal{A}} \hat{f}(A) \Psi_{A}\left(\eta^{B}\right)=\sum_{A \subset B} \sum_{C \subset A}(-1)^{\#(A \backslash C)} f\left(\eta^{C}\right)=f\left(\eta^{B}\right) .
$$

Therefore the equality of (3) is valid for every local function.

For a continuous function $f$ we can approximate it uniformly by a local function $f_{n}$ which is defined by

$$
f_{n}(\eta):=f\left(\eta_{\Lambda_{n}}\right)
$$

where

$$
\left(\eta_{\Lambda_{n}}\right)_{x}:= \begin{cases}\eta_{x} & \text { if } x \in \Lambda_{n}, \\ 0 & \text { if } x \notin \Lambda_{n},\end{cases}
$$

and $\Lambda_{n}$ is a cube centered at origin and of side $2 n+1$. Since $f_{n}$ is a local function, we can write

$$
f_{n}(\eta)=\sum_{A \in \mathcal{A}} \hat{f}_{n}(A) \Psi_{A}(\eta)
$$


It is not difficult to check that if $\bigcup_{i} A_{i} \subset \Lambda_{n}$ then $\hat{f}_{n}(A)=\hat{f}(A)$. Therefore (3) holds for every continuous function.

From now on, we regard the sequence $\{\hat{f}(A)\}_{A}$ as the coefficient of the continuous function of $f$ in the expansion (3).

We expand currents $w_{e}^{E}, w_{e}^{P}$ in the expansion (3). Then we have

$$
\begin{aligned}
& w_{e}^{E}=\sum_{i=1}^{k}\left\{c_{\mathrm{ex}}(i) i\left\{\Psi_{\emptyset 0, i}-\sum_{j=1}^{k} \Psi_{\emptyset^{0, i} ; e, j}\right\}\right\}-\sum_{i=1}^{k}\left\{c_{\mathrm{ex}}(i) i\left\{\Psi_{\emptyset e, i}-\sum_{j=1}^{k} \Psi_{\emptyset^{0}, j ; e, i}\right\}\right\} \\
& +\sum_{i=2}^{k} \sum_{j=1}^{k-1} c_{\mathrm{ge}}(i) \Psi_{\emptyset 0, i ; e, j}-\sum_{i=2}^{k} \sum_{j=1}^{k-1} c_{\mathrm{ge}}(i) \Psi_{\emptyset 0, i ; e, j}, \\
& w_{e}^{P}=\sum_{i=1}^{k}\left\{c_{\mathrm{ex}}(i)\left\{\Psi_{\emptyset^{0, i}}-\sum_{j=1}^{k} \Psi_{\emptyset^{0, i} ; e, j}\right\}\right\}-\sum_{i=1}^{k}\left\{c_{\mathrm{ex}}(i)\left\{\Psi_{\not \ell, i}-\sum_{j=1}^{k} \Psi_{\emptyset^{0, j ; e, i}}\right\}\right\},
\end{aligned}
$$

where $\emptyset^{x, i}, \emptyset^{x, i} ; y, j \in \mathcal{A}$ for $x, y \in \mathbf{Z}^{d}, x \neq y$ and $1 \leqslant i, j \leqslant k$ are defined respectively by

$$
\begin{aligned}
& \left(\emptyset^{x, i}\right)_{l}:= \begin{cases}\{x\} & \text { if } l=i, \\
\emptyset & \text { otherwise, }\end{cases} \\
& \left(\emptyset^{x, i ; y, j}\right)_{l}:= \begin{cases}\{x\} & \text { if } l=i \neq j, \\
\{y\} & \text { if } l=j \neq i, \\
\{x, y\} & \text { if } l=i=j, \\
\varnothing & \text { otherwise. }\end{cases}
\end{aligned}
$$

Denote by $\theta_{e}$ the reflection operator with respect to $1 / 2 e$ along the $e$ direction, namely for $x \in \mathbf{Z}^{d}$,

$$
\left(\theta_{e} x\right)_{e^{\prime}}:= \begin{cases}x_{e^{\prime}} & \text { if } e \neq e^{\prime} \\ -x_{e}+1 & \text { if } e=e^{\prime}\end{cases}
$$

We may extend $\theta_{e}$ to $\mathcal{A}$ naturally. Put

$$
\mathcal{B}_{e}:=\left\{\emptyset^{0, i}: 1 \leqslant i \leqslant k\right\} \cup\left\{\emptyset^{0, i ; e, j}: 1 \leqslant i, j \leqslant k\right\}
$$

and

$$
\begin{aligned}
& \tilde{w}_{e}^{E}(A):= \begin{cases}c_{\mathrm{ex}}(i) i & \text { if } A=\emptyset^{0, i} \text { for } 1 \leqslant i \leqslant k, \\
-c_{\mathrm{ex}}(i) i+c_{\mathrm{ge}}(i) & \text { if } A=\emptyset^{0, i ; e, j} \text { for } 2 \leqslant i \leqslant k, 1 \leqslant j \leqslant k-1, \\
-c_{\mathrm{ex}}(1) & \text { if } A=\emptyset^{0,1 ; e, j} \text { for } 1 \leqslant j \leqslant k, \\
-c_{\mathrm{ex}}(i) i & \text { if } A=\emptyset^{0, i ; e, k} \text { for } 2 \leqslant i \leqslant k, \\
0 & \text { otherwise, }\end{cases} \\
& \tilde{w}_{e}^{P}(A):= \begin{cases}c_{\mathrm{ex}}(i) & \text { if } A=\emptyset^{0, i} \text { for } 1 \leqslant i \leqslant k, \\
c_{\mathrm{ex}}(i) & \text { if } A=\emptyset^{0, i}, e, j \text { for } 1 \leqslant i, j \leqslant k, \\
0 & \text { otherwise, }\end{cases}
\end{aligned}
$$

where $\emptyset^{x, i}$ and $\emptyset^{x, i ; y, j}$ are defined by (7). In view of (6), it holds that

$$
\begin{aligned}
w_{e}^{E} & =\sum_{A \in \mathcal{B}_{e}} \tilde{w}_{e}^{E}(A)\left(\Psi_{A}-\Psi_{\theta_{e} A}\right), \\
w_{e}^{P} & =\sum_{A \in \mathcal{B}_{e}} \tilde{w}_{e}^{P}(A)\left(\Psi_{A}-\Psi_{\theta_{e} A}\right) .
\end{aligned}
$$


We define $A^{x \rightarrow y}$ and $A^{(x, y)}$ by

$$
\begin{aligned}
A^{x \rightarrow y}: & =\left\{\begin{array}{l}
\left(A_{1}, \ldots, A_{i-1} \cup\{x\}, A_{i} \backslash\{x\}, \ldots, A_{j} \backslash\{y\}, A_{j-1} \cup\{y\}, \ldots, A_{k}\right), \\
\text { if } x \in A_{i}, y \in A_{j} \text { such that } 2 \leqslant i \leqslant k, 1 \leqslant j \leqslant k-1, \\
A, \quad \text { otherwise, }
\end{array}\right. \\
A^{(x, y)}: & =\left\{\begin{array}{l}
\left(A_{1}, \ldots, A_{i} \backslash\{x\} \cup\{y\}, \ldots, A_{k}\right), \quad \text { if } x \in A_{i}, y \notin \bigcup_{j} A_{j} \text { such that } 1 \leqslant i \leqslant k, \\
A, \quad \text { otherwise. }
\end{array}\right.
\end{aligned}
$$

Given $x, y \in \mathbf{Z}^{d}$, define $\Upsilon_{x, y} \subset X$ by

$$
\Upsilon_{x, y}:=\left\{\eta: \eta_{x} \geqslant 2,1 \leqslant \eta_{y} \leqslant k-1\right\} .
$$

Then $\Psi_{A}\left(\eta^{x \rightarrow y}\right)=\Psi_{A^{y \rightarrow x}}(\eta)$ for $\eta \in \Upsilon_{x, y}$. Therefore using summation by parts formula, we get

$$
\begin{aligned}
\pi^{x \rightarrow y} f(\eta) & =1_{\left\{\eta_{x} \geqslant 2,1 \leqslant \eta_{y} \leqslant k-1\right\}}(\eta) \sum_{A} \hat{f}(A)\left(\Psi_{A^{y \rightarrow x}}(\eta)-\Psi_{A}(\eta)\right) \\
& =1_{\left\{\eta_{x} \geqslant 2,1 \leqslant \eta_{y} \leqslant k-1\right\}}(\eta) \sum_{A}\left(\hat{f}\left(A^{x \rightarrow y}\right)-\hat{f}(A)\right) \Psi_{A}(\eta) .
\end{aligned}
$$

For $A \in \mathcal{A}$, if $\Psi_{A}(\eta) \neq 0$ for some $\eta \in \Upsilon_{x, y}^{c}$, then $A^{x \rightarrow y}=A$. Therefore

$$
\pi^{x \rightarrow y} f(\eta)=\sum_{A}\left(\hat{f}\left(A^{x \rightarrow y}\right)-\hat{f}(A)\right) \Psi_{A}(\eta)
$$

Similarly

$$
\pi^{(x, y)} f(\eta)=\sum_{A}\left(\hat{f}\left(A^{(x, y)}\right)-\hat{f}(A)\right) \Psi_{A}(\eta) .
$$

If we define $\hat{c}_{\mathrm{ge}}(x, A)$ and $\hat{c}_{\mathrm{ex}}(x, A)$ by

$$
\begin{aligned}
& \hat{c}_{\mathrm{ge}}(x, A):= \begin{cases}c_{\mathrm{ge}}(i) & \text { if } x \in A_{i} \text { for } 1 \leqslant i \leqslant k, \\
0 & \text { otherwise, }\end{cases} \\
& \hat{c}_{\mathrm{ex}}(x, A):= \begin{cases}c_{\mathrm{ex}}(i) & \text { if } x \in A_{i} \text { for } 1 \leqslant i \leqslant k, \\
0 & \text { otherwise, }\end{cases}
\end{aligned}
$$

then we can rewrite $L f$ in the form

$$
L f(\eta)=\sum_{A \in \mathcal{A}} \hat{L} \hat{f}(A) \Psi_{A}(\eta)
$$

where

$$
\hat{L} \hat{f}(A)=\sum_{x, y \in \mathbf{Z}^{d},|x-y|=1}\left\{\hat{c}_{\mathrm{ge}}(x, A)\left(\hat{f}\left(A^{x \rightarrow y}\right)-\hat{f}(A)\right)+\hat{c}_{\mathrm{ex}}(x, A)\left(\hat{f}\left(A^{(x, y)}\right)-\hat{f}(A)\right)\right\} .
$$

Let $X_{S}$ be a Markov process on $\mathcal{A}$ whose generator is $\hat{L}$ defined by (10) and $P_{A}$ a distribution of the Markov process starting from $A$. Then the process is equivalent to the original process which starts from the configuration $\eta^{A}$. Therefore the Markov process generated by $\hat{L}$ inherits the conserved quantities from the original process. Namely, if we define $\mathcal{A}_{i, j} \subset \mathcal{A}$ by

$$
\mathcal{A}_{i, j}:=\{A \in \mathcal{A}: \# A=i, \tilde{\#} A=j\},
$$

then $\mathcal{A}_{i, j}$ becomes the ergodic classes of the Markov process generated by $\hat{L}$. 
Let $X_{S}$ be a Markov process on $\mathcal{A}$ whose generator is $\hat{L}$ defined by (10) and $P_{A}$ the distribution of the Markov process starting from $A$. Then the process is equivalent to the original process which starts from the configuration $\eta^{A}$.

Let the sequence $\left\{\hat{g}_{\lambda}(A)\right\}_{A}$ be defined by

$$
\hat{g}_{\lambda}(A):=E_{A} \int_{0}^{\infty} \hat{f}\left(X_{S}\right) \mathrm{e}^{-\lambda s} \mathrm{~d} s
$$

for a local function $f$. Put

$$
g_{\lambda}(\eta):=\sum_{A} \hat{g}_{\lambda}(A) \Psi_{A}(\eta)
$$

Lemma 3.2. The function $g_{\lambda}$ is well-define and an element of $C_{0}(X)$. Furthermore this function is the solution of the resolvent equation

$$
\lambda g_{\lambda}-L g_{\lambda}=f
$$

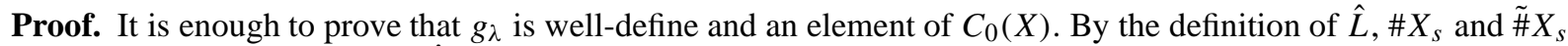
are conserved. By using (5), $\hat{f}(A)$ is zero if \#A or $\tilde{\#} A$ is large enough, and similarly for $\hat{g}_{\lambda}(A)$. We define the stopping time $\sigma_{n}$ by

$$
\sigma_{n}:= \begin{cases}\inf \left\{s: \bigcup_{i}\left(X_{s}\right)_{i} \subset \Lambda_{n}\right\} & \text { if }\left\{s: \bigcup_{i}\left(X_{s}\right)_{i} \subset \Lambda_{n}\right\} \neq \emptyset, \\ \infty & \text { if }\left\{s: \bigcup_{i}\left(X_{s}\right)_{i} \subset \Lambda_{n}\right\}=\emptyset,\end{cases}
$$

where $\Lambda_{n}$ is a cube centered at origin and of side $2 n+1$. Pick up a large $n$ such that if $\bigcup_{i} A_{i} \cap \Lambda_{n}^{c} \neq \emptyset$ then $\hat{f}(A)=0$. Therefore

$$
\hat{g}_{\lambda}(A)=E_{A}\left[\int_{\sigma_{n}}^{\infty} \hat{f}\left(X_{s}\right) \mathrm{e}^{-\lambda s} \mathrm{~d} s\right] \leqslant \frac{1}{\lambda} \hat{f}_{M} E_{A}\left[\mathrm{e}^{-\lambda \sigma_{n}}\right],
$$

where $\hat{f}_{M}=\max _{A} \hat{f}(A)$. We define $d_{n}(A)$ the sum of the supremum distances from $\Lambda_{n}$ to elements of $\bigcup_{i} A_{i}$ : formally if $\bigcup_{i} A_{i} \backslash \Lambda_{n}=\left\{x^{1}, \ldots, x^{m}\right\}$ then

$$
d_{n}(A):=\sum_{i=1}^{m}\left(\left|x^{i}\right|_{\infty}-n\right)
$$

Since the jump rate is bounded, it is not difficult to show that there exist constants $C_{1}, C_{2}>0$, which may depend on $\lambda$, such that

$$
E_{A}\left[\mathrm{e}^{-\lambda \sigma_{n}}\right] \leqslant C_{1} \mathrm{e}^{-C_{2} d_{n}(A)} .
$$

This shows that $g_{\lambda}(\eta)$ is well-define and an element of $C_{0}(X)$.

We give the reversible measure for $\hat{L}$, which we will use in Section 7. We define $m(A, p, \rho)$ by

$$
m(A, p, \rho):=E_{p, \rho}\left[\Psi_{A}\right],
$$

for $A \in \mathcal{A}, 0<p<1$ and $p<\rho<k p$. The discrete measure whose mass of $A$ is $m(A, p, \rho)$ is also denoted by $m$.

Lemma 3.3. For each $A \in \mathcal{A}, m$ is a smooth function of $p, \rho$, and for each pair of $p$ and $\rho, m$ is a reversible measure for $\hat{L}$. 
Remark. We decompose $\mathcal{A}$ into $\left\{\mathcal{A}_{i, j}\right\}$ the ergodic classes of the Markov process. On each ergodic class, $m(\cdot, p, \rho)$ for $0<p<1, p<\rho<k p$ are essentially the same. That is, for each ergodic class, $m(\cdot, p, \rho)$ and $m\left(\cdot, p^{\prime} \rho^{\prime}\right)$ are absolutely continuous each other and the Radon-Nikodym derivative is a constant depending only on $p, \rho, p^{\prime}, \rho^{\prime}$ and the ergodic class.

Proof of Lemma 3.3. We have only to prove that $m$ is a reversible measure for $\hat{L}$, or equivalently that

$$
\begin{array}{ll}
\hat{c}_{\mathrm{ge}}(x, A) m(A, p, \rho)=\hat{c}_{\mathrm{ge}}\left(y, A^{x \rightarrow y}\right) m\left(A^{x \rightarrow y}, p, \rho\right), & \text { for } A \neq A^{x \rightarrow y}, \\
\hat{c}_{\mathrm{ex}}(x, A) m(A, p, \rho)=\hat{c}_{\mathrm{ex}}\left(y, A^{(x, y)}\right) m\left(A^{(x, y)}, p, \rho\right), & \text { for } A \neq A^{(x, y)},
\end{array}
$$

for each $p, \rho$. But this relation immediately follows from the reversibility of the original process.

\section{Estimate of the expectation of occupation time in dimensions $d \geqslant 3$}

In this section we will estimate an expectation of occupation time for a certain set in dimensions $d \geqslant 3$.

Firstly we prove a general result on the expectation of occupation time for the Markov process.

Lemma 4.1. Let $\mathcal{B} \subset \mathcal{A}$, and suppose that there exist two subsets $\mathcal{B}_{1}, \mathcal{B}_{2}$ of $\mathcal{A}$ and functions $f_{1}$ and $f_{2}$ on $\mathcal{A}$ such that

$$
\begin{aligned}
& \mathcal{B} \subset \mathcal{B}_{1} \subset \mathcal{B}_{2}, \\
& f_{1} \geqslant 0, \\
& f_{1}(A) \geqslant 1 \quad \text { if } A \in \mathcal{B}_{1}, \\
& \hat{L} f_{1}(A) \leqslant 0 \quad \text { if } A \in \mathcal{B}_{1}^{c}, \\
& \sup _{A \in \mathcal{B}_{2}^{c}} f_{1}(A)<1, \\
& f_{2} \geqslant 0, \\
& \sup f_{2}<\infty, \\
& \hat{L} f_{2}(A) \leqslant-1 \quad \text { if } A \in \mathcal{B}_{2} .
\end{aligned}
$$

Then we have

$$
\sup _{A} \int_{0}^{\infty} E_{A}\left[1_{\mathcal{B}}\left(X_{S}\right)\right] \mathrm{d} s<\infty .
$$

Proof. We consider the martingale defined by

$$
M_{i, s}:=f_{i}\left(X_{s}\right)-f_{i}\left(X_{0}\right)-\int_{0}^{s} \hat{L} f_{i}\left(X_{u}\right) \mathrm{d} u,
$$

for $i=1,2$. Since $M_{i, 0}=0$, applying the Doob's optional sampling time theorem

$$
f_{1}(A)=E_{A}\left[f_{1}\left(X_{\sigma_{\mathcal{B}_{1}} \wedge N}\right)-\int_{0}^{\sigma_{\mathcal{B}_{1}} \wedge N} f_{1}\left(X_{u}\right) \mathrm{d} u\right]
$$


for all $N>0$, where $\sigma_{\mathcal{B}_{1}}$ is a first hitting time to $\mathcal{B}_{1}$. Substituting the conditions (15)-(17) the right-hand side is greater than

$$
E_{A}\left[I_{\left\{\sigma_{\mathcal{B}_{1}}<N\right\}}\right] .
$$

Therefore by condition (18)

$$
\sup _{A \in \mathcal{B}_{2}^{c}} P_{A}\left[\sigma_{\mathcal{B}_{1}}<\infty\right] \leqslant \sup _{A \in \mathcal{B}_{2}^{c}} f_{1}(A)<1 .
$$

Similarly, by using the conditions (19)-(21), we get

$$
\sup _{A \in \mathcal{B}_{2}} E_{A}\left[\sigma_{\mathcal{B}_{2}^{c}}\right] \leqslant \sup _{A \in \mathcal{B}_{2}} f_{2}(A)<\infty,
$$

where $\sigma_{\mathcal{B}_{2}^{c}}$ is the first hitting time to $\mathcal{B}_{2}^{c}$. On using the Markov property, the supremum of the expectation of occupation time for $\mathcal{B}$ is finite.

From now to the end of the section, we suppose that the dimensions $d$ is greater than or equal to 3 . In order to apply Lemma 4.1, we prepare some functions. We define functions $h_{1, l}, h_{2, l}$ from $\mathbf{Z}^{d}$ into $\mathbf{R}_{+}$, which are a small perturbation of the potential function on $\mathbf{Z}^{d}$, by

$$
\begin{aligned}
& h_{1, l}(x):= \begin{cases}1 & \text { if }|x| \leqslant l, \\
\left(\frac{1}{\sqrt{1+(|x|-l)^{2}}}\right)^{d-2} & \text { if }|x| \geqslant l,\end{cases} \\
& h_{2, l}(x):=\left(\frac{\sqrt{1+l}}{\sqrt{1+|x|^{2}}}\right)^{d-2},
\end{aligned}
$$

for $l \geqslant 0$, where $|\cdot|$ is the Euclidean norm of $\mathbf{Z}^{d}$. For $a=\left(a_{1}, a_{2}, a_{3}, a_{4}\right)$ such that $a_{1}, a_{2}, a_{3}>0$ and $a_{4} \geqslant 0$, we define a function $g_{a}$ from $\mathbf{R}_{+}$into $\mathbf{R}_{+}$satisfying the following conditions

- $g_{a}$ is a continuously differentiable function on $\mathbf{R}_{+}$.

- On the interval [0, $\left.a_{1}\right], g_{a}$ is a quadratic function with $-a_{2}$ for the coefficient of the second order term.

- There exists $b \geqslant a_{1}$ such that:

- On the interval $\left[a_{1}, b\right], g_{a}$ is a quadratic function with $a_{3}$ for the coefficient of the second order term.

- On the interval $[b, \infty), g_{a}$ is zero.

- The differential coefficient from the right at $x=0$ is $-a_{4}$.

Formally

$$
g_{a}(x):= \begin{cases}-a_{2}\left(x+\frac{a_{4}}{2 a_{2}}\right)^{2}+\frac{\left(2 a_{1} a_{2}+a_{4}\right)^{2}}{4}\left(\frac{1}{a_{3}}+\frac{1}{a_{2}}\right), & \text { if } 0 \leqslant x \leqslant a_{1}, \\ a_{3}\left\{x-\left(\frac{2 a_{1} a_{2}+a_{4}}{2 a_{3}}+a_{1}\right)\right\}^{2}, & \text { if } a_{1} \leqslant x \leqslant a_{1}+\frac{2 a_{1} a_{2}+a_{4}}{2 a_{3}}, \\ 0, & \text { if } x \geqslant a_{1}+\frac{2 a_{1} a_{2}+a_{4}}{2 a_{3}} .\end{cases}
$$

Let us define $\mathcal{A}_{M}$ by

$$
\mathcal{A}_{M}:=\{A \in \mathcal{A}: \# A=M\} .
$$

Assume that $\Lambda$ is a Euclidean ball with radius $l$, and define $\mathcal{B}:=\mathcal{B}_{M, l}$ by

$$
\mathcal{B}:=\left\{A \in \mathcal{A}_{M}: \bigcup A_{i} \cap \Lambda \neq \emptyset \text { or there exist } x, y \in \bigcup_{i} A_{i} \text { such that } 1 \leqslant|x-y|_{\infty} \leqslant l\right\} .
$$


Here $|\cdot|_{\infty}$ is the supremum norm of $\mathbf{Z}^{d}$. For $A \in \mathcal{A}_{M}$, we define $\left\{x_{i}: 1 \leqslant i \leqslant M\right\}:=\left\{x_{i}(A): 1 \leqslant i \leqslant M\right\} \subset \mathbf{Z}^{d}$ by

$$
\left\{x_{i}: 1 \leqslant i \leqslant M\right\}=\bigcup_{i} A_{i}
$$

and $x_{i, j}(A)$ by the $j$ th component of $x_{i}(A)$.

Let us define constants $a_{1}, a_{2}$ and $c^{ \pm}$by

$$
\begin{aligned}
& a_{1}:=\left(M^{2}+l, \frac{2}{c^{-}}, \frac{1}{c^{+} M^{2}}, 0\right), \\
& a_{2}:=\left(M^{2}+l, \frac{1}{c^{-}}, \frac{1}{2 c^{+} M^{2}}, \frac{4 M^{2} c^{+}}{c^{-}}\right),
\end{aligned}
$$

and

$$
\begin{aligned}
c^{-} & :=\min _{r} c_{\mathrm{ex}}(r), \\
c^{+} & :=\max _{r} c_{\mathrm{ex}}(r) .
\end{aligned}
$$

Lemma 4.2. Define sets $\mathcal{B}_{1}, \mathcal{B}_{2}$ and functions $f_{1}, f_{2}$ by

$$
\begin{aligned}
& \mathcal{B}_{1}:=\mathcal{B}, \\
& \mathcal{B}_{2}:=\left\{A \in \mathcal{A}_{M}: \text { there exists } x \in A \text { such that }|x|_{\infty} \leqslant M^{2}+l\right. \\
& \left.\quad \text { or there exists } x, y \in A \text { such that } 1 \leqslant|x-y|_{\infty} \leqslant M^{2}+l\right\}, \\
& f_{1}(A):=\sum_{i=1}^{M} h_{1, l}\left(x_{i}\right)+\sum_{1 \leqslant i<j \leqslant M} h_{2, l}\left(x_{i}-x_{j}\right), \\
& f_{2}(A):=\sum_{i=1}^{M} \sum_{j=1}^{d} g_{a_{1}}\left(\left|x_{i, j}\right|\right)+\sum_{1 \leqslant i<m \leqslant M} \sum_{j=1}^{d} g_{a_{2}}\left(\left|x_{i, j}-x_{m, j}\right|\right),
\end{aligned}
$$

where $a_{1}, a_{2}$ and $c^{ \pm}$are defined by (23)-(26). Then the conditions of Lemma 4.1 hold, namely (14)-(21) hold.

Corollary 4.3. Suppose that $d \geqslant 3$. Then for $\mathcal{B}$ defined by (22),

$$
\sup _{A} \int_{0}^{\infty} E_{A}\left[1_{\mathcal{B}}\left(X_{S}\right)\right] \mathrm{d} s<\infty .
$$

Proof of Lemma 4.2. We have only to check the truth of the conditions (17) and (21). We define $\mathcal{B}_{0}$ by

$$
\mathcal{B}_{0}=\mathcal{B}_{0, M}:=\left\{A \in \mathcal{A}_{M}:\left|x_{i}(A)-x_{j}(A)\right|>1 \text { for } i \neq j\right\} .
$$

Then on the set $\mathcal{B}_{0}, \hat{L}$ behave as a discrete Laplacian on $\mathbf{Z}^{d}$ for each $x_{i}(A)$. Formally, if we denote by $\Delta_{d}$ the discrete Laplacian on $\mathbf{Z}^{d}$, then

$$
\hat{L} f_{1}(A)=\sum_{i=1}^{M} \hat{c}_{\mathrm{ex}}\left(x_{i}(A), A\right)\left(\Delta_{d} h_{1, l}\right)\left(x_{i}\right)+\sum_{1 \leqslant i<m \leqslant M}\left(\hat{c}_{\mathrm{ex}}\left(x_{i}(A), A\right)+\hat{c}_{\mathrm{ex}}\left(x_{m}(A), A\right)\right)\left(\Delta_{d} h_{2, l}\right)\left(x_{i}-x_{m}\right),
$$

for $A \in \mathcal{B}_{0}$. Since $h_{1, l}, h_{2, l}$ are small perturbations of the $d$-dimensional potential function, each term of the righthand side is non-positive. Since $\mathcal{B}_{1}^{c} \subset \mathcal{B}_{0}$, the condition (17) is valid. 
We also have

$$
\begin{aligned}
\hat{L} f_{2}(A)= & \sum_{i=1}^{M} \sum_{j=1}^{d} \hat{c}_{\mathrm{ex}}\left(x_{i}(A), A\right)\left(\Delta_{1} g_{a_{1}}\right)\left(\left|x_{i, j}(A)\right|\right) \\
& +\sum_{1 \leqslant i<m \leqslant M} \sum_{j=1}^{d}\left(\hat{c}_{\mathrm{ex}}\left(x_{i}(A), A\right)+\hat{c}_{\mathrm{ex}}\left(x_{m}(A), A\right)\right)\left(\Delta_{1} g_{a_{1}}\right)\left(\left|x_{i, j}(A)-x_{m, j}(A)\right|\right),
\end{aligned}
$$

for $A \in \mathcal{B}_{0}$. From the choice of the second component of $a_{1}, a_{2}$, if $\left|x_{i, j}(A)\right| \leqslant M^{2}+l$, then

$$
\hat{c}_{\mathrm{ex}}\left(x_{i}(A), A\right)\left(\Delta_{1} g_{a_{1}}\right)\left(\left|x_{i, j}(A)\right|\right) \leqslant-2
$$

and if $2 \leqslant\left|x_{i, j}(A)-x_{m, j}(A)\right| \leqslant M^{2}+l$, then

$$
\left(\hat{c}_{\mathrm{ex}}\left(x_{i}(A), A\right)+\hat{c}_{\mathrm{ex}}\left(x_{m}(A), A\right)\right)\left(\Delta_{1} g_{a_{1}}\right)\left(\left|x_{i, j}(A)-x_{m, j}(A)\right|\right) \leqslant-2 .
$$

From the choice of the third component of $a_{1}, a_{2}$, each terms of the last equality is less than or equal to $1 / M^{2}$. On the set $\mathcal{B}_{2} \cap \mathcal{B}_{0}$, there exists $i$ such that $\left|x_{i, j}(A)\right| \leqslant M^{2}+l$ for all $j$, or there exists $i, m$ such that $2 \leqslant \mid x_{i, j}(A)-$ $x_{m, j}(A) \mid \leqslant M^{2}+l$ for all $j$. Therefore on the set $\mathcal{B}_{2} \cap \mathcal{B}_{0}$, the condition (21) is valid.

On the set $\mathcal{B}_{2} \backslash \mathcal{B}_{0}$, without loss of generality we assume that there exist $n \geqslant 2$ and $1 \leqslant j \leqslant d$ such that $x_{l}=$ $x_{1}+(l-1) e_{j}$ for $1 \leqslant l \leqslant n$, where $e_{j}$ is a unit vector for the $j$ th coordinate, and if $n+1 \leqslant i \leqslant M$, then $\left|x_{i}-x_{m}\right| \geqslant 2$ for all $1 \leqslant m \leqslant M, i \neq m$. Furthermore, we can assume that the choice of the $x_{i}\left(A^{(x, y)}\right)$ is similar to $x_{i}(A)$, namely we assume that

$$
x_{i}\left(A^{(y, z)}\right)= \begin{cases}x_{i}(A) & \text { if } y \neq x_{i}(A), \\ z & \text { if } y=x_{i}(A),\end{cases}
$$

for all $1 \leqslant i \leqslant M, y \in \bigcup_{i} A_{i}$ and $z \notin \bigcup_{i} A_{i}$. Then $\hat{L} g_{a_{1}}\left(\left|x_{i, j}(A)\right|\right)$ and $\hat{L} g_{a_{2}}\left(\left|x_{i, j}(A)-x_{m, j}(A)\right|\right)$ make sense for $1 \leqslant i<m \leqslant M$. By elementary computation if $1 \leqslant i<m \leqslant n$, then

$$
\hat{L} g_{a_{2}}\left(\left|x_{i, j}(A)-x_{m, j}(A)\right|\right) \leqslant 0
$$

and

$$
\begin{aligned}
& \hat{L} g_{a_{2}}\left(\left|x_{1, j}(A)-x_{2, j}(A)\right|\right) \leqslant-\frac{4 c^{+} M^{2}}{c^{-}}, \\
& \hat{L} g_{a_{2}}\left(\left|x_{n-1, j}(A)-x_{n, j}(A)\right|\right) \leqslant-\frac{4 c^{+} M^{2}}{c^{-}} .
\end{aligned}
$$

The last value $\frac{4 c^{+} M^{2}}{c^{-}}$is given by the choice of the fourth component of $a_{2}$. We also compute that

$$
\begin{aligned}
\hat{L} \sum_{1 \leqslant i \leqslant n} g_{a_{1}}\left(\left|x_{i, j}(A)\right|\right)= & \hat{c}_{\mathrm{ex}}\left(x_{1}(A), A\right)\left\{g_{a_{1}}\left(\left|x_{1, j}(A)-1\right|\right)-g_{a_{1}}\left(\left|x_{1, j}(A)\right|\right)\right\} \\
& +\hat{c}_{\mathrm{ex}}\left(x_{n}(A), A\right)\left\{g_{a_{1}}\left(\left|x_{n, j}(A)+1\right|\right)-g_{a_{1}}\left(\left|x_{n, j}(A)\right|\right)\right\} .
\end{aligned}
$$

Since we assume that $x_{i, j}(A)=x_{i-1, j}(A)+1$ for $2 \leqslant i \leqslant n$, the right-hand side of the last equality is equal to

$$
\begin{aligned}
& \hat{c}_{\mathrm{ex}}\left(x_{1}(A), A\right) \sum_{1 \leqslant i \leqslant n}\left(\Delta_{1} g_{a_{1}}\right)\left(\left|x_{i, j}(A)\right|\right) \\
& \quad+\left\{\hat{c}_{\mathrm{ex}}\left(x_{n}(A), A\right)-\hat{c}_{\mathrm{ex}}\left(x_{1}(A), A\right)\right\}\left\{g_{a_{1}}\left(\left|x_{n, j}(A)+1\right|\right)-g_{a_{1}}\left(\left|x_{n, j}(A)\right|\right)\right\} .
\end{aligned}
$$

From the choice of the third component of $a_{1}$, the first line is less than or equal to $m / M^{2}$. Since the maximal value of $g_{a_{1}}(x)-g_{a_{1}}(x+1)$ is given by the maximal value of differential coefficient of $g_{a_{1}}$, from the choice of $a_{1}$ the second line is less than or equal to $4 c^{+}\left(M^{2}+l\right) / c^{-}$. From the assumption of the $x_{i}$ for $i \geqslant n+1$, the other terms for $\hat{L} f_{2}$ is less than or equal to $1 / M^{2}$. Thus we have checked the truth of the condition (21). 


\section{Occupation time for some stochastic processes}

In this section, we consider finite state continuous time stochastic jump processes, which may not be Markovian.

Let $S$ be a finite set, which is a state space of our stochastic process. For each $i \in S$, there is given a probability measure $\mu^{i}$ on $S \times \mathbf{R}_{+}$. Let $\left(Y^{i, n}, \sigma^{i, n}\right), i \in S, n=0,1,2, \ldots$ be a system of independent random variables taking values in $S \times \mathbf{R}_{+}$such that for each $i$ the joint distribution of $\left(Y^{i, n}, \sigma^{i, n}\right)$ is given by $\mu^{i}$ for every $n$.

Then our process is described as follows. Suppose that the process starts at $i_{0} \in S$. Then one takes the random variable $\left(Y^{i_{0}, 0}, \sigma^{i_{0}, 0}\right)$ and let the process stay at $i_{0}$ up to the time $\sigma^{i_{0}, 0}$ and jump to $Y^{i_{0}, 0}$ at time $\sigma^{i_{0}, 0}$. The procedure is repeated over by letting it start at $i_{1}=Y^{i_{0}, 0}$ and taking up $\left(Y^{i_{1}, 1}, \sigma^{i_{1}, 1}\right)$ place of $\left(Y^{i_{0}, 0}, \sigma^{i_{0}, 0}\right)$. We denote the distribution of the process which starts from $i \in S$ by $P_{i}$. We suppose several conditions for the joint distribution $\mu^{i}$. Let us define

$$
F_{i, j}(\lambda):=E^{\mu_{i}}\left[1_{\left\{Y^{i}=j\right\}} \mathrm{e}^{-\lambda \sigma^{i}}\right]
$$

(A.1) It holds that

$$
F_{i, i}(\lambda)=0
$$

for all $i \in S$.

(A.2) There exist constants $p_{i, j}, f_{i, j}$, and a function $g(\lambda)$ such that

$$
F_{i, j}(\lambda)=p_{i, j}-f_{i, j} g(\lambda)+\mathrm{o}(g(\lambda))
$$

where $p_{i, j} \geqslant 0$ and for all $i \in S$

$$
\sum_{j \in S} p_{i, j}=1
$$

$f_{i, j}$ is non-negative; and $g(\lambda)$ is a positive and increasing function from $\mathbf{R}_{+}$to $\mathbf{R}_{+}$which vanishes at $\lambda=0$. Here $\mathrm{o}(\cdot)$ is Landau's symbol as $\lambda$ tends to 0 . In this paper $g(\lambda)$ will be $\sqrt{\lambda}$ or $-1 / \log \lambda$ accordingly as $d=1$ or 2 .

(A.3) The stochastic matrix $p_{i, j}$ in (A.2) is irreducible.

(A.4) There exists a pair $(i, j)$ such that $f_{i, j}$ is strictly positive.

(A.5) There exists $i_{0}$ such that $f_{i_{0}, j}=0$ for all $j$. Furthermore, there exists a positive constant $f_{i_{0}}$ such that

$$
\sum_{j} F_{i_{0}, j}(\lambda)=1-f_{i_{0}} \lambda+o(\lambda) \text {. }
$$

Lemma 5.1. Suppose that (A.1)-(A.5) hold. Then for all $i \in S$, the Laplace transform of occupation time for $i_{0}$ is asymptotically equivalent to $1 / g(\lambda)$ (as $\lambda$ tends to 0 ), namely there exists a constant $C_{1}$ which depends on $i$ such that

$$
E_{i} \int_{0}^{\infty} 1_{\left\{i_{0}\right\}}\left(X_{s}\right) \mathrm{e}^{-\lambda s} \mathrm{~d} s=\frac{C_{1}}{g(\lambda)}+\mathrm{o}\left(\frac{1}{g(\lambda)}\right) .
$$

Furthermore for all $i, j \in S$, there exists a limit of the difference of Laplace transform of occupation time for $i_{0}$, namely there exists a constant $C_{2}$ which depends on $i$ and $j$ such that

$$
\lim _{\lambda \rightarrow 0}\left\{E_{i} \int_{0}^{\infty} 1_{\left\{i_{0}\right\}}\left(X_{s}\right) \mathrm{e}^{-\lambda s} \mathrm{~d} s-E_{j} \int_{0}^{\infty} 1_{\left\{i_{0}\right\}}\left(X_{s}\right) \mathrm{e}^{-\lambda s} \mathrm{~d} s\right\}=C_{2} .
$$

Proof. Let $Q_{i, i_{0}}(\lambda)$ be the Laplace transform of the occupation time for $i_{0}$ with starting point $i \in S$. Let $\sigma_{1}$ be the first jump time. Then on using the conditional independence of the process after the time $\sigma_{1}$ and the jump time $\sigma_{1}$ given $X_{\sigma_{1}}$, 


$$
\begin{aligned}
Q_{i, i_{0}}(\lambda) & =\delta_{i, i_{0}} E_{i_{0}} \int_{0}^{\sigma_{1}} \mathrm{e}^{-\lambda s} \mathrm{~d} s+\sum_{j} E_{i} \mathrm{e}^{-\lambda \sigma_{1}} 1_{\left\{X_{\sigma_{1}}=j\right\}} E_{j} \int_{0}^{\infty} 1_{\left\{i_{0}\right\}}\left(X_{s}\right) \mathrm{e}^{-\lambda s} \mathrm{~d} s \\
& =\delta_{i, i_{0}} \frac{1}{\lambda}\left(1-\sum_{j} F_{i_{0}, j}(\lambda)\right)+\sum_{j} F_{i, j}(\lambda) Q_{j, i_{0}}(\lambda) .
\end{aligned}
$$

Therefore $Q_{i, i_{0}}(\lambda)$ is given by

$$
Q_{i, i_{0}}(\lambda)=\left((I-F(\lambda))^{-1} x(\lambda)\right)_{i},
$$

where

$$
(x(\lambda))_{i}=\delta_{i, i_{0}} \frac{1}{\lambda}\left(1-\sum_{j} F_{i_{0}, j}(\lambda)\right) .
$$

Recall that by (A.5), $\frac{1}{\lambda}\left(1-\sum_{j} F_{i_{0}, j}(\lambda)\right)=f_{i_{0}}+\mathrm{o}(1)$.

Let $(\widetilde{I-p})_{i, j}$ be $(i, j)$-element of $I-p$ cofactor matrix. Then it is easy to see

$$
\operatorname{det}(I-F(\lambda))=\operatorname{det}(I-p)+\sum_{i, j} f_{i, j}(\widetilde{I-p})_{i, j} g(\lambda)+\mathrm{o}(g(\lambda)) .
$$

Since $p$ is a stochastic matrix $\operatorname{det}(I-p)=0$. Since $p$ is irreducible, $(\widetilde{I-p})_{i, j}>0$ for all $i, j \in S$. Using the conditions (A.2) and (A.4), we get $\sum_{i, j} f_{i, j}(\widetilde{I-p})_{i, j}>0$. Put $C_{0}:=\sum_{i, j} f_{i, j}(\widetilde{I-p})_{i, j}$, then

$$
(\operatorname{det}(I-F(\lambda)))^{-1}=\frac{1}{C_{0} g(\lambda)}+\mathrm{o}\left(\frac{1}{g(\lambda)}\right) .
$$

Furthermore there exists a matrix $C_{i, j}$ such that cofactor matrix of $I-F(\lambda)$ is given by

$$
(\widetilde{I-F(\lambda)})_{i, j}=(\widetilde{I-p})_{i, j}+C_{i, j} g(\lambda)+\mathrm{o}(g(\lambda)) .
$$

Therefore

$$
Q_{i, i_{0}}(\lambda)=\frac{(\widetilde{I-p})_{i, i_{0}} f_{i_{0}}}{C_{0}} \frac{1}{g(\lambda)}+\mathrm{o}\left(\frac{1}{g(\lambda)}\right) .
$$

Since $p_{i, j}$ is a stochastic matrix that is irreducible we see that $\widetilde{(I-p)_{i, j}}=(\widetilde{I-p})_{k, j}$ for all $i, j, k \in S$. Since the dependence of $Q_{i, i_{0}}(\lambda)$ on $i$ comes from $(\widetilde{I-F(\lambda)})_{i, l}$ whose principal term cancels by the subtraction of $Q_{j, i_{0}}(\lambda)$,

$$
\lim _{\lambda \rightarrow 0}\left(Q_{i, i_{0}}(\lambda)-Q_{j, i_{0}}(\lambda)\right)=f_{i_{0}} C_{0}\left(C_{i, i_{0}}-C_{j, i_{0}}\right) .
$$

On using the result of the discrete time random walk (see for example [7]) we can easily get following results.

Proposition 5.2. Let us consider continuous time symmetric simple random walk on $\mathbf{Z}$ with the jump rate $r$. Then there exists a constant $C=C(r)$ such that

$$
E_{1}\left[\mathrm{e}^{-\lambda \mu_{\{0\}}}\right]=1-C \sqrt{\lambda}+\mathrm{o}(\sqrt{\lambda}),
$$

where $\mu_{\{0\}}$ is a first hitting time to $\{0\}$.

Proposition 5.3. Let us consider continuous time symmetric simple random walk on $\mathbf{Z}^{2}$, and $B:=\left\{0, \pm e_{1}, \pm e_{2}\right\}$ where $e_{1}, e_{2}$ are positive unit vector on $\mathbf{Z}^{2}$. Then there exists a positive constants $p_{x, y}, C_{x, y}$ which depends on the 
starting point $x \in \mathbf{Z}^{2}$, the first visit point to $B, y \in B$ say, and the coefficient of the exponential holding time such that

$$
E_{x}\left[e^{-\lambda \mu_{B}} 1_{\left\{X_{\mu_{B}}=y\right\}}\right]=p_{x, y}+C_{x, y} \frac{1}{\log \lambda}+\mathrm{o}\left(\frac{1}{\log \lambda}\right),
$$

with

$$
\sum_{y \in B \backslash\{0\}} p_{x, y}=1,
$$

for $x \notin B$, where $\mu_{B}$ is a first hitting time to $B$.

\section{Estimate of the expectation of occupation time}

In this section $X_{S}$ is a Markov process on $\mathcal{A}$ whose generator is $\hat{L}$ defined by (10), $P_{A}$ a distribution of the Markov process starting from $A, \mathcal{A}_{i, j}$ the ergodic classes of the Markov process defined by (11), and $\theta_{e}$ a reflection operator defined via (8).

Let $e_{1}, e_{2}, \ldots, e_{d}$ be positive unit vector on $\mathbf{Z}^{d}$ and $\Lambda=\{0,1\}$ for $d=1$ and $\Lambda=\left\{0, e_{1}, \ldots, e_{d}\right\}$ for $d \geqslant 2$. Let us define $\mathcal{D}_{1}, \mathcal{D}_{2}^{e}, \mathcal{D}_{2}$ by

$$
\begin{aligned}
& \mathcal{D}_{1}:=\left\{\emptyset^{0, i}: 1 \leqslant i \leqslant k\right\}, \\
& \mathcal{D}_{2}^{e}:=\left\{\emptyset^{0, i ; e, j}: 1 \leqslant i, j \leqslant k\right\}, \\
& \mathcal{D}_{2}:= \begin{cases}\mathcal{D}_{2}^{1} & \text { for } d=1, \\
\bigcup_{l=1}^{d} \mathcal{D}_{2}^{e_{l}} & \text { for } d \geqslant 2 .\end{cases}
\end{aligned}
$$

For $B \in \mathcal{D}_{1} \cup \mathcal{D}_{2}$ we also define $\mathcal{B}(B)$ by

$$
\mathcal{B}(B):=\left\{A \in \mathcal{A}: \text { there exists } x \in \mathbf{Z}^{d} \text { such that }\left(\tau_{x} A\right)_{i} \cap \Lambda=B_{i} \text { for all } i\right\} .
$$

Lemma 6.1. Suppose $C \in \mathcal{D}_{1}$. Then

$$
E_{C} \int_{0}^{\infty} 1_{\mathcal{B}(B)}\left(X_{S}\right) \mathrm{e}^{-\lambda s} \mathrm{~d} s-E_{\theta_{e} C} \int_{0}^{\infty} 1_{\mathcal{B}(B)}\left(X_{S}\right) \mathrm{e}^{-\lambda s} \mathrm{~d} s=0
$$

for all $B \in \mathcal{D}_{1} \cup \mathcal{D}_{2}$.

Proof. Since $C \in \mathcal{D}_{1}$, there exists $1 \leqslant i \leqslant k$ such that $C=\emptyset^{0, i}$. Then $\theta_{e} C=\emptyset^{e, i}$. Therefore $C$ and $\theta_{e} C$ are in the same ergodic class $\mathcal{A}_{1, i}$. It is easy to see that if $B \in \mathcal{A}_{1, i}$ then $\mathcal{B}(B)=\mathcal{A}_{1, i}$ and if $B \notin \mathcal{A}_{1, i}$ then $\mathcal{B}(B) \cap \mathcal{A}_{1, i}=\emptyset$. Therefore

$$
E_{C} \int_{0}^{\infty} 1_{\mathcal{B}(B)}\left(X_{s}\right) \mathrm{e}^{-\lambda s} \mathrm{~d} s=E_{\theta_{e} C} \int_{0}^{\infty} 1_{\mathcal{B}(B)}\left(X_{s}\right) \mathrm{e}^{-\lambda s} \mathrm{~d} s=\frac{1}{\lambda}
$$

for $B \in \mathcal{A}_{1, i}$ and

$$
E_{C} \int_{0}^{\infty} 1_{\mathcal{B}(B)}\left(X_{S}\right) \mathrm{e}^{-\lambda s} \mathrm{~d} s=E_{\theta_{e} C} \int_{0}^{\infty} 1_{\mathcal{B}(B)}\left(X_{S}\right) \mathrm{e}^{-\lambda s} \mathrm{~d} s=0
$$

for $B \notin \mathcal{A}_{1, i}$. 
For $C \in \mathcal{D}_{2}$, we define $\mathcal{D}(C)$ by

$$
\mathcal{D}(C):=\left\{B \in \mathcal{D}_{1}: \tilde{\#} C-k \leqslant \tilde{\#} B \leqslant \tilde{\#} C-1\right\} \cup\left\{B \in \mathcal{D}_{2}: \tilde{\#} B=\tilde{\#} C\right\} .
$$

Lemma 6.2. Suppose $C \in \mathcal{D}_{2}$ and $B \in\left(\mathcal{D}_{1} \cup \mathcal{D}_{2}\right) \backslash \mathcal{D}(C)$. Then it holds that

$$
E_{C} \int_{0}^{\infty} 1_{\mathcal{B}(B)}\left(X_{S}\right) \mathrm{e}^{-\lambda s} \mathrm{~d} s=E_{\theta_{e} C} \int_{0}^{\infty} 1_{\mathcal{B}(B)}\left(X_{S}\right) \mathrm{e}^{-\lambda s} \mathrm{~d} s=0 .
$$

Proof. By the definition of $\mathcal{D}(C)$ it is clear that if $B \in\left(\mathcal{D}_{1} \cap \mathcal{D}_{2}\right) \backslash \mathcal{D}(C)$, then $\mathcal{B}(B) \cap \mathcal{A}(C)=\emptyset$, where $\mathcal{A}(C)$ is the set of ergodic classes which include the element $C$. It is also clear that $\mathcal{D}(C)=\mathcal{D}\left(\theta_{e} C\right)$ and $\mathcal{A}(C)=\mathcal{A}\left(\theta_{e} C\right)$. Therefore it holds that

$$
E_{C} \int_{0}^{\infty} 1_{\mathcal{B}(B)}\left(X_{s}\right) \mathrm{e}^{-\lambda s} \mathrm{~d} s=E_{\theta_{e} C} \int_{0}^{\infty} 1_{\mathcal{B}(B)}\left(X_{s}\right) \mathrm{e}^{-\lambda s} \mathrm{~d} s=0
$$

Lemma 6.3. Suppose $C \in \mathcal{D}_{2}$ and $B \in \mathcal{D}(C) \cap \mathcal{D}_{1}$. Then there exists a subset $\left\{D_{l}(B)\right\}_{l=1}^{d} \subset \mathcal{D}(C) \cap \mathcal{D}_{2}$, which is defined by (28) below, such that

$$
\begin{aligned}
& E_{C} \int_{0}^{\infty} 1_{\mathcal{B}(B)}\left(X_{S}\right) \mathrm{e}^{-\lambda s} \mathrm{~d} s-E_{\theta_{e} C} \int_{0}^{\infty} 1_{\mathcal{B}(B)}\left(X_{s}\right) \mathrm{e}^{-\lambda s} \mathrm{~d} s \\
& \quad=\sum_{l=1}^{d}\left\{-E_{C} \int_{0}^{\infty} 1_{\mathcal{B}\left(D_{l}(B)\right)}\left(X_{s}\right) \mathrm{e}^{-\lambda s} \mathrm{~d} s+E_{\theta C} \int_{0}^{\infty} 1_{\mathcal{B}\left(D_{l}(B)\right)}\left(X_{s}\right) \mathrm{e}^{-\lambda s} \mathrm{~d} s\right\} .
\end{aligned}
$$

Proof. Since $C \in \mathcal{D}_{2}$, without loss of generality we suppose $C \in \mathcal{A}_{2, j}$ for some $2 \leqslant j \leqslant 2 k$ in this proof. Since $B \in \mathcal{D}_{1}$, there exists $1 \leqslant i \leqslant k$ such that $B=\emptyset^{0, i}$. Then

$$
\mathcal{B}(B) \cap \mathcal{A}_{2, j}= \begin{cases}\left\{\emptyset^{x, i ; y, j-i}: x, y \in \mathbf{Z}^{d}, y-x \neq 0, e_{1}, e_{2}, \ldots, e_{d}\right\} & \text { if } 1 \leqslant j-i \leqslant k, \\ \emptyset & \text { otherwise. }\end{cases}
$$

Let us define $\mathcal{A}_{2, j}^{i} \subset \mathcal{A}_{2, j}$ by

$$
\mathcal{A}_{2, j}^{i}:=\left\{A=\left(A_{1}, A_{2}, \ldots, A_{k}\right) \in \mathcal{A}_{2, j}: A_{i} \neq \emptyset\right\} .
$$

Then it is easy to check that

$$
\mathcal{B}(B) \cap \mathcal{A}_{2, j} \subset \mathcal{A}_{2, j}^{i} .
$$

It is convenient to introduce the difference set

$$
\mathcal{B}_{2, j}^{\prime}(B):=\mathcal{A}_{2, j}^{i} \backslash\left(\mathcal{B}(B) \cap \mathcal{A}_{2, j}\right) .
$$

It is not difficult to check that

$$
\mathcal{B}_{2, j}^{\prime}(B)=\left\{\emptyset^{x, i ; y, j-i}: x, y \in \mathbf{Z}^{d}, y-x=e_{1}, e_{2}, \ldots, e_{d}\right\}=\bigcup_{l=1}^{d} \mathcal{B}\left(\emptyset^{0, i ; e_{l}, j-i}\right) .
$$

Set

$$
D_{l}(B)=D_{l}\left(\emptyset^{0, i}\right):=\emptyset^{0, i ; e_{l}, j-i} \in \mathcal{D}_{2} .
$$


Then we have

$$
E_{C} \int_{0}^{\infty} 1_{\mathcal{B}(B)}\left(X_{s}\right) \mathrm{e}^{-\lambda s} \mathrm{~d} s=E_{C} \int_{0}^{\infty}\left(1_{\mathcal{A}_{2, j}^{i}}\left(X_{s}\right)-\sum_{l=1}^{d} 1_{\mathcal{B}\left(D_{l}(B)\right)}\left(X_{s}\right)\right) \mathrm{e}^{-\lambda s} \mathrm{~d} s .
$$

Since our Markov process and $\mathcal{A}_{2, j}^{i}$ are mirror symmetric,

$$
E_{C} \int_{0}^{\infty} 1_{\mathcal{A}_{2, j}^{i}}\left(X_{S}\right) \mathrm{e}^{-\lambda s} \mathrm{~d} s=E_{\theta C} \int_{0}^{\infty} 1_{\mathcal{A}_{2, j}^{i}}\left(X_{S}\right) \mathrm{e}^{-\lambda s} \mathrm{~d} s
$$

Therefore we have

$$
\begin{aligned}
& E_{C} \int_{0}^{\infty} 1_{\mathcal{B}(B)}\left(X_{S}\right) \mathrm{e}^{-\lambda s} \mathrm{~d} s-E_{\theta_{e} C} \int_{0}^{\infty} 1_{\mathcal{B}(B)}\left(X_{s}\right) \mathrm{e}^{-\lambda s} \mathrm{~d} s \\
& \quad=\sum_{l=1}^{d}\left\{-E_{C} \int_{0}^{\infty} 1_{\mathcal{B}\left(D_{l}(B)\right)}\left(X_{S}\right) \mathrm{e}^{-\lambda s} \mathrm{~d} s+E_{\theta C} \int_{0}^{\infty} 1_{\mathcal{B}\left(D_{l}(B)\right)}\left(X_{S}\right) \mathrm{e}^{-\lambda s} \mathrm{~d} s\right\} .
\end{aligned}
$$

Lemma 6.4. Suppose $C \in \mathcal{D}_{2}$ and $B \in \mathcal{D}(C) \cap \mathcal{D}_{2}$. Then there exists a constant $C_{1}$ which depends on $B, C$ and dimension $d$ such that

$$
\lim _{\lambda \rightarrow 0}\left\{E_{C} \int_{0}^{\infty} 1_{\mathcal{B}(B)}\left(X_{s}\right) \mathrm{e}^{-\lambda s} \mathrm{~d} s-E_{\theta_{e} C} \int_{0}^{\infty} 1_{\mathcal{B}(B)}\left(X_{s}\right) \mathrm{e}^{-\lambda s} \mathrm{~d} s\right\}=C_{1} .
$$

Further there exists a positive constant $C_{2}$ which depends on $B, C$ and the dimension d such that

$$
E_{C} \int_{0}^{\infty} 1_{\mathcal{B}(B)}\left(X_{s}\right) \mathrm{e}^{-\lambda s} \mathrm{~d} s= \begin{cases}\frac{C_{2}}{\sqrt{\lambda}}+\mathrm{o}\left(\frac{1}{\sqrt{\lambda}}\right), & \text { if } d=1, \\ -C_{2} \log \lambda+\mathrm{o}(\log \lambda), & \text { if } d=2, \\ C_{2}+\mathrm{o}(1), & \text { if } d \geqslant 3 .\end{cases}
$$

Here $\mathrm{o}(\cdot)$ is a Landau's symbol as $\lambda$ tends to 0 .

Proof. Firstly we prove the lemma for $d \geqslant 3$. If we substitute 2 for $l$ in (22), then $\mathcal{B}(B)$ is a subset of $\mathcal{B}$ defined by (22). By using Corollary 4.3 , we have already proved that

$$
E_{C} \int_{0}^{\infty} I_{\mathcal{B}(B)}\left(X_{s}\right) \mathrm{d} s<\infty
$$

Therefore we conclude the proof of this lemma for $d \geqslant 3$.

Secondly we prove the lemma for $d=1$. The proof is divided into 2 steps.

Step 1 . Since $C \in \mathcal{D}_{2}$, without loss of generality we suppose $C \in \mathcal{A}_{2, j}$ for some $2 \leqslant j \leqslant 2 k$ in this proof. Since $B$ is an element of $\mathcal{D}(C) \cap \mathcal{D}_{2}$, there exists $i$ such that $1 \leqslant i, j-i \leqslant k$ and $B=\emptyset^{0, i ; 1, j-i}$. Then $\mathcal{B}(B)$ may be written as

$$
\mathcal{B}(B)=\left\{\emptyset^{x, i ; x+1, j-i}: x \in \mathbf{Z}\right\} .
$$

We can also rewrite $\mathcal{A}_{2, j}$ as

$$
\mathcal{A}_{2, j}=\left\{\emptyset^{x, m ; y, j-m}: x, y \in \mathbf{Z}, x \neq y, 1 \leqslant m, j-m \leqslant k\right\} .
$$


Let us consider the equivalence relation $\sim$ in $\mathcal{A}_{2, j}$ which is defined by

$$
\emptyset^{x, m ; y, j-m} \sim \emptyset^{x^{\prime}, m^{\prime} ; y^{\prime}, j-m^{\prime}} \quad \text { if and only if } \quad x-y=x^{\prime}-y^{\prime}, \text { and } m=m^{\prime},
$$

the quotient set of $\mathcal{A}_{2, j}$ relative to relation $\sim$, and representative. Since $\emptyset^{0, m ; l, j-m} \sim \emptyset^{0, j-m ;-l, m}$, we can take the representative in the form $\emptyset^{0, m ; l, j-m}$ for $1 \leqslant m, j-m \leqslant k$ and $l \in \mathbf{N}$. It is convenient to denote $\emptyset^{0, m ; l, j-m}$ by $(l, m)$. Since $\emptyset^{0, m ; l, j-m}$ means that there exist two particle at 0 and $l$ with energy $m$ and $j-m$ respectively, the value $l$ and $m$ means that the distance of two particles and the number of energy which the left particle carries, respectively. It is easy to see that if $B \in \mathcal{A}_{2, j}$ and $A \in \mathcal{B}(B) \cap \mathcal{A}_{2, j}$ then $A \sim B$. Since our process is shift invariant, we project our process with respect to the relation $\sim$, namely we consider the following.

Let $\mathcal{X}_{j}$ be the set of representative of the quotient set of $\mathcal{A}_{2, j}$ relative to $\sim$ : formally

$$
\mathcal{X}_{j}:=\{(l, m): l \in \mathbf{N}, m \in \mathbf{Z} \text { with } 1 \leqslant m, j-m \leqslant k\} .
$$

The process is a continuous time random walk on $\mathcal{X}_{j}$ with the jump rate given by

$$
r\left((l, m),\left(l^{\prime}, m^{\prime}\right)\right)= \begin{cases}c_{\mathrm{ex}}(m)+c_{\mathrm{ex}}(j-m) & \text { if }\left|l-l^{\prime}\right|=1 \text { and } m=m^{\prime}, \\ c_{\mathrm{ge}}(m) & \text { if } l=l^{\prime}=1 \text { and } m-m^{\prime}=1, \\ c_{\mathrm{ge}}(j-m) & \text { if } l=l^{\prime}=1 \text { and } m-m^{\prime}=-1, \\ 0 & \text { otherwise. }\end{cases}
$$

Namely the state space has $\min \{j-1, k\}-\max \{j-k, 1\}+1$ half lines which are linearly ordered. On each half line except the point 1 , the process evolves as a symmetric simple random walk with some constant jump rate (the constant may depend on the half line). Each half line is connected to the next and previous half lines at point 1 . At this point the random walk is not symmetric. We denote the distribution of the random walk which starts at $\mathbf{x} \in \mathcal{X}_{j}$ by $\mathbf{P}_{\mathbf{x}}$. Then

$$
E_{C} \int_{0}^{\infty} 1_{\mathcal{B}(B)}\left(X_{S}\right) \mathrm{e}^{-\lambda s} \mathrm{~d} s=\mathbf{E}_{\mathbf{c}} \int_{0}^{\infty} 1_{\{\mathbf{b}\}}\left(\mathbf{X}_{s}\right) \mathrm{e}^{-\lambda s} \mathrm{~d} s,
$$

where $B=\emptyset^{0, n ; 1, j-n}, C=\emptyset^{x, l ; x+m, j-l}, \mathbf{b}=(1, n)$ and $\mathbf{c}=(m, l)$.

Step 2. In this step we prove that there exists $C_{1}$ which depends on $\mathbf{b}, \mathbf{c} \in \mathcal{X}_{j}$ such that

$$
\mathbf{E}_{\mathbf{c}} \int_{0}^{\infty} 1_{\{\mathbf{b}\}}\left(\mathbf{X}_{s}\right) \mathrm{e}^{-\lambda s} \mathrm{~d} s=\frac{C_{1}}{\sqrt{\lambda}}+\mathrm{o}\left(\frac{1}{\sqrt{\lambda}}\right)
$$

but there exists a limit of the difference

$$
\lim _{\lambda \rightarrow 0}\left\{\mathbf{E}_{\mathbf{c}_{1}} \int_{0}^{\infty} 1_{\{\mathbf{b}\}}\left(\mathbf{X}_{s}\right) \mathrm{e}^{-\lambda s} \mathrm{~d} s-\mathbf{E}_{\mathbf{c}_{2}} \int_{0}^{\infty} 1_{\{\mathbf{b}\}}\left(\mathbf{X}_{s}\right) \mathrm{e}^{-\lambda s} \mathrm{~d} s\right\},
$$

which depends on $\mathbf{b}, \mathbf{c}_{1}, \mathbf{c}_{2} \in \mathcal{X}_{j}$.

Since we know the distribution of the first hitting time of 0 for the continuous time symmetric simple random walk on $\mathbf{Z}$ with rate $r$ starting at 1 , we treat $\{(l, m): m \geqslant 2\} \in \mathcal{X}_{j}$ as one point for each $l$. Formally we consider for each $j$ the following the continuous time stochastic process which is not Markovian. The state space is $\mathcal{Y}_{j}$ defined by

$$
\mathcal{Y}_{j}:=\{(l, m): l \in\{1,2\}, m \in \mathbf{Z}, 1 \leqslant m, j-m \leqslant k\} .
$$


The value $l$ means that if the distance of two particles is 1 then $l=1$ and otherwise $l=2$, and the value of $m$ indicates the number of energy carried by the left particle. Define $\left\{F_{\left(l_{1}, m_{1}\right),\left(l_{2}, m_{2}\right)}(\lambda)\right\}_{\left(l_{1}, m_{1}\right),\left(l_{2}, m_{2}\right) \in \mathcal{Y}_{j}}$ by

$$
F_{\left(l_{1}, m_{1}\right),\left(l_{2}, m_{2}\right)}(\lambda):= \begin{cases}H\left(\lambda ; c_{\mathrm{ex}}\left(m_{1}\right)+c_{\mathrm{ex}}\left(j-m_{1}\right)\right) & \text { if } l_{1}=2, l_{2}=1, m_{1}=m_{2}, \\ G\left(\lambda ; Z_{m_{1}}\right) \frac{c_{\mathrm{ex}}\left(m_{1}\right)+c_{\mathrm{ex}}\left(j-m_{1}\right)}{Z_{m_{1}}} & \text { if } l_{1}=1, l_{2}=2, m_{1}=m_{2}, \\ G\left(\lambda ; Z_{m_{1}}\right) \frac{c_{\mathrm{ge}}\left(m_{1}\right)}{Z_{m_{1}}} & \text { if } l_{1}=l_{2}=1, m_{1}-m_{2}=1, \\ G\left(\lambda ; Z_{m_{1}}\right) \frac{c_{\mathrm{ge}}\left(j-m_{1}\right)}{Z_{m_{1}}} & \text { if } l_{1}=l_{2}=1, m_{1}-m_{2}=-1, \\ 0 & \text { otherwise, }\end{cases}
$$

where $Z_{m}=Z_{m, j}:=c_{\mathrm{ex}}(m)+c_{\mathrm{ex}}(j-m)+c_{\mathrm{ge}}(m)+c_{\mathrm{ge}}(j-m)$ and $H(\cdot ; r)$ and $G(\cdot ; r)$ are the Laplace transform of the first hitting time of 0 for the continuous time symmetric simple random walk on $\mathbf{Z}$ with rate $r$ starting at 1 and the Laplace transform of the exponential distribution with rate $r$, respectively. Our stochastic process is the same as that defined in Section 5 with $\left\{F_{\left(l_{1}, m_{1}\right),\left(l_{2}, m_{2}\right)}(\lambda)\right\}_{\left(l_{1}, m_{1}\right),\left(l_{2}, m_{2}\right) \in \mathcal{Y}_{j}}$ in place of $F_{i, j}(\lambda)$. Let us denote the process and the distribution of the stochastic process which starts at $\mathbf{x} \in \mathcal{Y}_{j}$ by $\mathbf{Y}_{s}$ and $\tilde{\mathbf{P}}_{\mathbf{x}}$, respectively. Then it holds that

$$
\mathbf{E}_{\mathbf{c}} \int_{0}^{\infty} 1_{\{\mathbf{b}\}}\left(\mathbf{X}_{s}\right) \mathrm{e}^{-\lambda s} \mathrm{~d} s=\tilde{\mathbf{E}}_{\mathbf{x}} \int_{0}^{\infty} 1_{\{\mathbf{y}\}}\left(\mathbf{Y}_{s}\right) \mathrm{e}^{-\lambda s} \mathrm{~d} s,
$$

where $\mathbf{b}=(1, l), \mathbf{c}=(1, n) \in \mathcal{X}_{j}$ and $\mathbf{x}=(1, l), \mathbf{y}=(1, n) \in \mathcal{Y} j$ for all $1 \leqslant l, j-l, n, j-n \leqslant k$. We can apply Lemma 5.1 with the help of Proposition 5.2 and get that there exist $C_{1}$ and $C_{2}$ which depend on $\mathbf{x}_{1}, \mathbf{x}_{2}$ and $\mathbf{y}$ such that

$$
\begin{aligned}
& \tilde{\mathbf{E}}_{\mathbf{x}_{1}} \int_{0}^{\infty} 1_{\{\mathbf{y}\}}\left(\mathbf{Y}_{s}\right) \mathrm{e}^{-\lambda s} \mathrm{~d} s=\frac{C_{1}}{\sqrt{\lambda}}+\mathrm{o}\left(\frac{C_{1}}{\sqrt{\lambda}}\right), \\
& \lim _{\lambda \rightarrow 0}\left\{\tilde{\mathbf{E}}_{\mathbf{x}_{1}} \int_{0}^{\infty} 1_{\{\mathbf{y}\}}\left(\mathbf{Y}_{s}\right) \mathrm{e}^{-\lambda s} \mathrm{~d} s-\tilde{\mathbf{E}}_{\mathbf{x}_{2}} \int_{0}^{\infty} 1_{\{\mathbf{y}\}}\left(\mathbf{Y}_{s}\right) \mathrm{e}^{-\lambda s} \mathrm{~d} s\right\}=C_{2},
\end{aligned}
$$

where $\mathbf{x}_{1}=\left(l_{1}, 1\right), \mathbf{x}_{2}=\left(l_{2}, 1\right), \mathbf{y}=(n, 1) \in \mathcal{Y}_{j}$ for all $1 \leqslant l_{1}, j-l_{1}, l_{2}, j-l_{2}, n, j-n \leqslant k$. Therefore we conclude the proof for this lemma for $d=1$.

The proof of this lemma for $d=2$ is similar to that for $d=1$. We indicate what we need to modify.

Step 1. Let $\mathcal{X}_{j}^{2}$ be the state space of our new Markov process defined by

$$
\mathcal{X}_{j}^{2}:=\left\{(l, m): l \in \mathbf{Z}^{2} \backslash\{\boldsymbol{0}\}, m \in \mathbf{Z} \text { with } 1 \leqslant m, j-m \leqslant k\right\} .
$$

The values $l$ and $m$ means the difference of two particles and the number of energy on which the datums particle carries, respectively. The process is a continuous time random walk on $\mathcal{X}_{j}^{2}$ with jump rate $r\left((l, m),\left(l^{\prime}, m^{\prime}\right)\right)$ as

$$
r\left((l, m),\left(l^{\prime}, m^{\prime}\right)\right)= \begin{cases}c_{\mathrm{ex}}(m)+c_{\mathrm{ex}}(j-m) & \text { if }\left|l-l^{\prime}\right|=1 \text { and } m=m^{\prime}, \\ c_{\mathrm{ge}}(m) & \text { if } l=l^{\prime},|l|=1 \text { and } m-m^{\prime}=1, \\ c_{\mathrm{ge}}(j-m) & \text { if } l=l^{\prime},|l|=1 \text { and } m-m^{\prime}=-1, \\ 0 & \text { otherwise. }\end{cases}
$$

Namely the state space has ordered $\min \{j-1, k\}-\max \{j-k, 1\}+12$-dimensional lattice planes without origin. On planes the process is symmetric simple random walk with some jump rate. Each planes is connected to the next and previous planes at points $\pm e_{1}$ and $\pm e_{2}$. At this point the random walk is not symmetric. We write the distribution of the random walk which starts from $\mathbf{x} \in \mathcal{X}_{j}^{2}$ by $\mathbf{P}_{\mathbf{x}}$. Since $\emptyset^{x, l ; y, j-l}=\emptyset^{y, j-l ; x, l} \in \mathcal{A}_{2, j} \mathcal{X}_{j}^{2}$ is not the 
representative of the quotient set of $\mathcal{A}_{2, j}$ relative to $\sim$. But we suppose that the point $\emptyset^{0, l ; x, j-l} \in \mathcal{A}_{2, j}$ is branches $(x, l)$ and $(-x, j-l) \in \mathcal{X}_{j}^{2}$. Then following is truth. Suppose $B=\emptyset^{0, n ; e, j-n}, C=\emptyset^{x, l ; x+m, j-l} \in \mathcal{A}_{2, j}, \mathbf{b}_{1}=$ $(e, n), \mathbf{b}_{2}=(-e, j-n)$ and $\mathbf{c}=(m, l) \in \mathcal{X}_{j}^{2}$. Then it holds that

$$
E_{C} \int_{0}^{\infty} 1_{\mathcal{B}(B)}\left(X_{S}\right) \mathrm{e}^{-\lambda s} \mathrm{~d} s=\mathbf{E}_{\mathbf{c}} \int_{0}^{\infty}\left(1_{\left\{\mathbf{b}_{1}\right\}}\left(\mathbf{X}_{s}\right)+1_{\left\{\mathbf{b}_{2}\right\}}\left(\mathbf{X}_{s}\right)\right) \mathrm{e}^{-\lambda s} \mathrm{~d} s .
$$

Therefore we conclude step 1.

Step 2. What we have to prove is that there exists $C_{1}$ which depends on $\mathbf{b}, \mathbf{c} \in \mathcal{X}_{j}^{2}$ such that

$$
\mathbf{E}_{\mathbf{c}} \int_{0}^{\infty} 1_{\{\mathbf{b}\}}\left(\mathbf{X}_{s}\right) \mathrm{e}^{-\lambda s} \mathrm{~d} s=-C_{1} \log \lambda+\mathrm{o}(\log \lambda)
$$

and there exists a limit of the difference

$$
\lim _{\lambda \rightarrow 0}\left\{\mathbf{E}_{\mathbf{c}_{1}} \int_{0}^{\infty} 1_{\{\mathbf{b}\}}\left(\mathbf{X}_{s}\right) \mathrm{e}^{-\lambda s} \mathrm{~d} s-\mathbf{E}_{\mathbf{c}_{2}} \int_{0}^{\infty} 1_{\{\mathbf{b}\}}\left(\mathbf{X}_{s}\right) \mathrm{e}^{-\lambda s} \mathrm{~d} s\right\} .
$$

We consider following continuous time stochastic process which is not Markov process, similar to that for $d=1$. Let $\mathcal{Y}_{j}^{2}$ be a state space of the process defined by

$$
\mathcal{Y}_{j}^{2}:=\left\{(l, m): l \in \mathbf{Z}^{2},\|l\|=1 \text { or } 2, m \in \mathbf{Z}, 1 \leqslant m, j-m \leqslant k\right\},
$$

where $\|l\|=\left|l_{1}\right|+\left|l_{2}\right|$ for $l=\left(l_{1}, l_{2}\right) \in \mathbf{Z}^{2}$. Put $\{F\}$ by

$$
F_{\left(l_{1}, m_{1}\right),\left(l_{2}, m_{2}\right)}(\lambda):= \begin{cases}H^{2}\left(\lambda ; c_{\mathrm{ex}}\left(m_{1}\right)+c_{\mathrm{ex}}\left(j-m_{1}\right), l_{1}, l_{2}\right) & \text { if }\left\|l_{1}\right\|=2,\left\|l_{2}\right\|=1, m_{1}=m_{2}, \\ G\left(\lambda ; Z_{m_{1}}\right) \frac{3\left(c_{\mathrm{ex}}\left(m_{1}\right)+c_{\mathrm{ex}}\left(j-m_{1}\right)\right)}{Z_{m_{1}}} & \text { if }\left\|l_{1}\right\|=1,\left\|l_{1}-l_{2}\right\|=1, m_{1}=m_{2}, \\ G\left(\lambda ; Z_{m_{1}}\right) \frac{c_{\mathrm{ge}}\left(m_{1}\right)}{Z_{m_{1}}} & \text { if } l_{1}=l_{2},\left\|l_{1}\right\|=1, m_{1}-m_{2}=1, \\ G\left(\lambda ; Z_{m_{1}}\right) \frac{c_{\mathrm{ge}}\left(j-m_{1}\right)}{Z_{m_{1}}} & \text { if } l_{1}=l_{2},\left\|l_{1}\right\|=1, m_{1}-m_{2}=-1, \\ 0 & \text { otherwise, }\end{cases}
$$

where $Z_{m}=Z_{m, j}:=3\left(c_{\mathrm{ex}}(m)+c_{\mathrm{ex}}(j-m)\right)+c_{\mathrm{ge}}(m)+c_{\mathrm{ge}}(j-m)$ and $G(\lambda ; r)$ is the Laplace transform of the exponential distribution with rate $r$, and $H^{2}(\lambda ; r, x, y)$ is defined as follows. Let $B=\left\{0, \pm e_{1}, \pm e_{2}\right\} \subset \mathbf{Z}^{2}$. Denote by $X^{r}$ and $P_{x}$ the continuous time symmetric simple random walk on $\mathbf{Z}^{2}$ with jump rate $r$ and the distribution of the walk which starts from $x \in \mathbf{Z}^{2}$. Let $\mu$ be the first hitting time to $B$. Then we define $H^{2}(\lambda ; r, x, y)$ by

$$
H^{2}(\lambda ; r, x, y):=E_{x}\left[\mathrm{e}^{-\lambda \mu} 1_{\left\{X_{\mu}^{r}=y\right\}}\right] .
$$

Comparing this process and that for $d=1$, the site $(1, m) \in \mathcal{Y}_{j}$ corresponds to $(l, m) \in \mathcal{Y}_{j}^{2}$, with $\|l\|=1$ and $(2, m) \in \mathcal{Y}_{j}$ corresponds to $(l, m) \in \mathcal{Y}_{j}^{2}$, with $\|l\|=2$. Denote by $\mathbf{Y}_{s}$ and $\tilde{\mathbf{P}}_{\mathbf{x}}$ the process and the distribution of the stochastic process which starts from $\mathbf{x} \in \mathcal{Y}_{j}$ respectively. Then it holds that

$$
\mathbf{E}_{\mathbf{c}} \int_{0}^{\infty} 1_{\{\mathbf{b}\}}\left(\mathbf{X}_{s}\right) \mathrm{e}^{-\lambda s} \mathrm{~d} s=\tilde{\mathbf{E}}_{\mathbf{x}} \int_{0}^{\infty} 1_{\{\mathbf{y}\}}\left(\mathbf{Y}_{s}\right) \mathrm{e}^{-\lambda s} \mathrm{~d} s,
$$

where $\mathbf{b}=(e, l), \mathbf{c}=\left(e^{\prime}, n\right) \in \mathcal{X}_{j}$ and $\mathbf{x}=(e, l), \mathbf{y}=\left(e^{\prime}, n\right) \in \mathcal{Y} j$ for all $1 \leqslant l, j-l, n, j-n \leqslant k$ and $e, e^{\prime} \in \mathbf{Z}^{2}$ with $\|e\|=\left\|e^{\prime}\right\|=1$. We can apply Lemma 5.1 with the help of Proposition 5.3 and get that there exist $C_{1}$ and $C_{2}$ which depend on $\mathbf{x}_{1}, \mathbf{x}_{2}$ and $\mathbf{y}$ such that 


$$
\begin{aligned}
& \tilde{\mathbf{E}}_{\mathbf{x}_{1}} \int_{0}^{\infty} 1_{\{\mathbf{y}\}}\left(\mathbf{Y}_{s}\right) \mathrm{e}^{-\lambda s} \mathrm{~d} s=-C_{1} \log \lambda+\mathrm{o}(\log \lambda), \\
& \lim _{\lambda \rightarrow 0}\left\{\tilde{\mathbf{E}}_{\mathbf{x}_{1}} \int_{0}^{\infty} 1_{\{\mathbf{y}\}}\left(\mathbf{Y}_{s}\right) \mathrm{e}^{-\lambda s} \mathrm{~d} s-\tilde{\mathbf{E}}_{\mathbf{x}_{2}} \int_{0}^{\infty} 1_{\{\mathbf{y}\}}\left(\mathbf{Y}_{s}\right) \mathrm{e}^{-\lambda s} \mathrm{~d} s\right\}=C_{2},
\end{aligned}
$$

where $\mathbf{x}_{1}=\left(l_{1}, 1\right), \mathbf{x}_{2}=\left(l_{2}, 1\right), \mathbf{y}=(n, 1) \in \mathcal{Y}_{j}$ for all $1 \leqslant l_{1}, j-l_{1}, l_{2}, j-l_{2}, n, j-n \leqslant k$. Thus the proof for $d=2$ is complete.

\section{Proof of theorems}

Firstly we prove Theorem 2.2.

Proof of Theorem 2.2. We define $\Lambda=\Lambda_{d} \subset \mathbf{Z}^{d}$ by

$$
\begin{aligned}
& \Lambda_{1}:=\{0,1\}, \\
& \Lambda_{d}:=\left\{0, e_{1}, e_{2}, \ldots, e_{d}\right\}, \quad \text { if } d \geqslant 2,
\end{aligned}
$$

where $e_{1}, e_{2}, \ldots, e_{d}$ are positive unit vector on $\mathbf{Z}^{d}$. We recall that $\mathcal{W}$ is the linear space spanned by the currents $w_{e}^{E}, w_{e}^{P}$ for all positive unit vectors $e$. Then for any $f \in \mathcal{W}, f$ depends only on $\left\{\eta_{x}: x \in \Lambda\right\}$, and

$$
E_{p, \rho}\left[f \mid \mathcal{F}_{\Lambda}\right]=0
$$

for all $p, \rho$, where $\mathcal{F}_{\Lambda}$ is $\sigma$-algebra generated by $\sum_{x \in \Lambda} 1_{\left\{\eta_{x} \neq 0\right\}}, \sum_{x \in \Lambda} \eta_{x}$ and $\left\{\eta_{x}: x \notin \Lambda\right\}$.

In [3], Kipnis and Varadhan proved some equivalent relation about central limit theorem variance. We use one of them. It holds that

$$
\int_{0}^{\infty} \sum_{x} E_{p, \rho}\left[f \tau_{x} \mathrm{e}^{L t} f\right] \mathrm{d} t=\lim _{\lambda \rightarrow 0} \sum_{x} E_{p, \rho}\left[f \tau_{x} g_{\lambda}\right],
$$

where $g_{\lambda}$ is a solution of the resolvent equation,

$$
\lambda g_{\lambda}-L g_{\lambda}=f \text {. }
$$

It is convenient to write $F_{\lambda}(p, \rho)=\sum_{x} E_{p, \rho}\left[f \tau_{x} g_{\lambda}\right]$. By Lemma 3.2 we have

$$
F_{\lambda}(p, \rho)=\sum_{x, A: \bigcup_{i}\left(\tau_{x} A\right)_{i} \cap \Lambda \neq \emptyset} \hat{g}_{\lambda}(A) E_{p, \rho}\left[f \Psi_{\tau_{x} A}\right]+\sum_{x, A: \bigcup_{i}\left(\tau_{x} A\right)_{i} \cap \Lambda=\emptyset} \hat{g}_{\lambda}(A) E_{p, \rho}\left[f \Psi_{\tau_{x} A}\right],
$$

where $\hat{g}_{\lambda}$ is defined by (12). By (30), the second sum on the right-hand side of (31) vanishes. We define the function $h$ by

$$
h(B, p, \rho):=\frac{E_{p, \rho}\left[f \Psi_{B}\right]}{m(B, p, \rho)}
$$

for $B \in \mathcal{A}$ such that $\bigcup_{i} B_{i} \subset \Lambda$. We recall that

$$
\mathcal{B}(B):=\left\{A \in \mathcal{A} \text { : there exists } x \in \mathbf{Z}^{d} \text { such that }\left(\tau_{x} A\right)_{i} \cap \Lambda=B_{i} \text { for all } i\right\}
$$

for $B \in \mathcal{A}$, such that $\bigcup_{i} B_{i} \subset \Lambda$, which is defined in Section 6. Then the first sum on the right-hand side of (31) is equal to

$$
\sum_{B \in \mathcal{A}: \cup_{i} B_{i} \subset \Lambda} \sum_{A \in \mathcal{B}(B)} h(B, p, \rho) \hat{g}_{\lambda}(A) m(A, p, \rho) .
$$


We substitute (12) and use the reversibility of $m$ to see that $F_{\lambda}$ is equal to

$$
\sum_{B \in \mathcal{A}: \bigcup_{i} B_{i} \subset \Lambda} h(B, p, \rho) \sum_{C \in \mathcal{A}} \hat{f}(C) m(C, p, \rho) E_{C} \int_{0}^{\infty} 1_{\mathcal{B}(B)}\left(X_{S}\right) \mathrm{e}^{-\lambda s} \mathrm{~d} s .
$$

In view of (9), there exists $\left\{\tilde{f}_{e}(C)\right\}$ which is a linear combination of $\left\{\tilde{w}_{e}^{E}(C), \tilde{w}_{e}^{P}(C)\right\}$ such that

$$
\begin{aligned}
F_{\lambda}(p, \rho)= & \sum_{B \in \mathcal{A}: \cup_{i} B_{i} \subset \Lambda} h(B, p, \rho) \sum_{C \in \mathcal{A}} \tilde{f}_{e}(C) m(C, p, \rho) \\
& \times\left\{E_{C} \int_{0}^{\infty} 1_{\mathcal{B}(B)}\left(X_{s}\right) \mathrm{e}^{-\lambda s} \mathrm{~d} s-E_{\theta C} \int_{0}^{\infty} 1_{\mathcal{B}(B)}\left(X_{s}\right) \mathrm{e}^{-\lambda s} \mathrm{~d} s\right\} .
\end{aligned}
$$

By (9), we know that $\tilde{f}_{e}(C)=0$ if $C \notin \mathcal{D}_{1} \cup \mathcal{D}_{2}$, and

$$
\left\{B \in \mathcal{A}: \bigcup_{i} B_{i} \subset \Lambda\right\}=\mathcal{D}_{1} \cup \mathcal{D}_{2},
$$

where $\mathcal{D}_{1}$ and $\mathcal{D}_{2}$ are defined by (27). Therefore we have

$$
\begin{aligned}
F_{\lambda}(p, \rho)= & \sum_{B \in \mathcal{D}_{1} \cup \mathcal{D}_{2}} h(B, p, \rho) \sum_{C \in \mathcal{D}_{1} \cup \mathcal{D}_{2}} \tilde{f}(C) m(C, p, \rho) \\
& \times\left\{E_{C} \int_{0}^{\infty} 1_{\mathcal{B}(B)}\left(X_{S}\right) \mathrm{e}^{-\lambda s} \mathrm{~d} s-E_{\theta C} \int_{0}^{\infty} 1_{\mathcal{B}(B)}\left(X_{S}\right) \mathrm{e}^{-\lambda s} \mathrm{~d} s\right\} .
\end{aligned}
$$

We have decomposed $F_{\lambda}(p, \rho)$ into a finite sum of smooth functions such that each term of it is the product of two factors, one is a smooth function of $p, \rho$ which does not depend on $\lambda$, and the other is a function of $\lambda$ which does not depend on $p, \rho$. Furthermore the second one is given by a difference of expectation of Laplace transform of occupation time for certain infinite set. Applying Lemmas 6.1-6.4, each of the second one converges as $\lambda$ tends to 0 . Therefore the limiting function of $F_{\lambda}(p, \rho)$ is also smooth.

Proof of Theorem 2.1. Firstly we claim that

$$
\begin{aligned}
(a \cdot \tilde{D}(p, \rho) a)= & E_{p, \rho}\left[c_{\mathrm{ex}}\left(\eta_{0}\right)\left\{\pi^{(0, e)}\left(a_{1} \eta_{0}+a_{2} 1_{\left\{\eta_{0} \neq 0\right\}}\right\}^{2}\right]\right. \\
& +E_{p, \rho}\left[c_{\mathrm{ge}}\left(\eta_{0}\right)\left\{\pi^{0 \rightarrow e}\left(a_{1} \eta_{0}+a_{2} 1_{\left\{\eta_{0} \neq 0\right\}}\right)\right\}^{2}\right]-\frac{1}{2} \int_{0}^{\infty} \sum_{x} E_{p, \rho}\left[w_{a} \tau_{x} \mathrm{e}^{L t} w_{a}\right] \mathrm{d} t
\end{aligned}
$$

for each $0<p<1, p<\rho<k p$, where

$$
w_{a}:=a_{1} w_{e}^{E}+a_{2} w_{e}^{P} .
$$

The proof of the equivalence of the variational formula (given by (1)) for the diffusion coefficient matrix and that by Green-Kubo formula (right-hand side of (33)) can be carried out in a way similar to that indicated for the proof of Proposition 2.2 of [8], p. 180. Therefore we omit the detail.

Secondly we show that there exists a smooth extension of $D$. Firstly we treat the diffusion coefficient matrix $D$ as a function of $p, \alpha$, namely we take expectations with respect to $v_{p, \alpha}$. Then all expectations for local functions are polynomials of $p$ and rational functions of $\alpha$. Put 


$$
\begin{aligned}
& \beta(\alpha):=\frac{1}{Z_{\alpha}}\left\{1+\sum_{l=2}^{k} l \frac{\alpha^{l-2}}{c_{\mathrm{ge}}(2) c_{\mathrm{ge}}(3) \cdots c_{\mathrm{ge}}(l)}\right\}, \\
& \gamma(\alpha):=\frac{1}{Z_{\alpha}}\left\{1+\sum_{l=2}^{k} l^{2} \frac{\alpha^{l-2}}{c_{\mathrm{ge}}(2) c_{\mathrm{ge}}(3) \cdots c_{\mathrm{ge}}(l)}\right\} .
\end{aligned}
$$

Then the inverse matrix of $\chi=\chi(p, \rho)$ defined by (2) is equal to

$$
\frac{1}{p\left(\gamma-\beta^{2}\right)}\left(\begin{array}{cc}
1 & -\beta \\
-\beta & \frac{\gamma-p \beta^{2}}{1-p}
\end{array}\right) .
$$

By the definition, $Z_{\alpha}$ is a polynomial of $\alpha$. Furthermore, the constant term of $Z_{\alpha}$ is not zero. We also have the following: there exists a polynomial $\Gamma_{0}(\alpha)$ whose constant term is not zero such that $\gamma-\beta^{2}=\alpha \Gamma_{0} / Z_{\alpha}^{2}$. By (32) and the definition of $m,(a \cdot \tilde{D} a)$ is a polynomial of $p$ and rational function of $\alpha$ for each two dimensional vector $a$. Furthermore there exist a positive integer $r$ and a polynomial $\Gamma_{1}^{a}(p, \alpha)$ whose constant term is not zero such that $(a \cdot \tilde{D} a)=p \alpha \Gamma_{1}^{a} / Z_{\alpha}^{r}$. Therefore we have a smooth extension of $D$ as a function of $p, \alpha$ at $p=0$ or $\alpha=0$. Since $\rho$ is a smooth and strictly increasing function of $p$ and $\alpha$, we have a smooth extension of $D$ as a function of $p$ and $\rho$ at $p=0$ or $\rho=p$ (corresponding to $p=0$ or $\alpha=0$ respectively). Using the same method we also have a smooth extension at $p=1$ or $\rho=k p$.

\section{Acknowledgements}

I would like to thank Professor Kôhei Uchiyama for all his useful suggestions and help. I also would like to thank the editor of the Annales de l'Institut Henri Poincaré, Probabilités et Statistiques and anonymous referees.

\section{References}

[1] C. Bernardin, Regularity of the diffusion coefficient for lattice gas reversible under Bernoulli measures, Stochastic Process. Appl. 101 (1) (2002) 43-68.

[2] C. Kipnis, C. Landim, S. Olla, Hydrodynamic limit for a nongradient system: the generalized symmetric exclusion process, Comm. Pure Appl. Math. 47 (11) (1994) 1475-1545.

[3] C. Kipnis, S.R.S. Varadhan, Central limit theorem for additive functionals of reversible Markov processes and applications to simple exclusions, Comm. Math. Phys. 104 (1) (1986) 1-19.

[4] C. Landim, S. Olla, S.R.S. Varadhan, Symmetric simple exclusion process: regularity of the self-diffusion coefficient, Comm. Math. Phys. 224 (1) (2001) 307-321.

[5] T.M. Liggett, Interacting Particle Systems, Springer, 1985.

[6] Y. Nagahata, Fluctuation dissipation equation for lattice gas with energy, J. Statist. Phys. 110 (1/2) (2003) 219-346.

[7] F. Spitzer, Principle of Random Walk, Nostrand, 1964.

[8] H. Spohn, Large Scale Dynamics of Interacting Particles, Springer, 1991. 